\title{
CANONICAL HEIGHTS FOR RANDOM ITERATIONS IN CERTAIN VARIETIES
}

\author{
SHU KAWAGUCHI
}

\begin{abstract}
We show the existence of canonical heights (normalized heights) of subvarieties for bounded sequences of morphisms and give some applications.
\end{abstract}

\section{INTRODUCTION}

Theory of heights of points (or subvarieties in general) is a basic tool in Diophantine geometry. Particular heights that enjoy nice properties, called "canonical heights" or "normalized heights," are sometimes of great use as are Néron-Tate heights on abelian varieties (see, for example, Hindry-Silverman [11, Part B, Part F.2]). On the other hand, dynamics of sequences of polynomial mappings (random iterations) in $\mathbb{P}^{N}$ over $\mathbb{C}$ have been studied in Russakovskii-Sodin [15] and Fornæss-Weickert [9] among others. In particular, in [9] invariant currents that transform well relative to such sequences and their average have been considered.

In this paper, we show the existence of heights of subvarieties that transform well relative to "bounded" sequences of such morphisms and give some applications. Although our interest mainly lies in morphisms of $\mathbb{P}^{N}$ of degree greater than or equal to 2 , our results also hold in the setting of Call-Silverman [7] and Zhang [20]: Namely, let $X$ be a projective variety over a number field $K$ and $L$ a line bundle on $X$, and we consider morphisms $f: X \rightarrow X$ such that $f^{*} L \simeq L^{\otimes d_{f}}$ for some integer $d_{f} \geq 2$.

Let $\boldsymbol{f}=\left(f_{i}\right)_{i=1}^{\infty}$ be a sequence of morphisms such that $f_{i}: X \rightarrow X$ satisfies $f_{i}^{*} L \simeq L^{\otimes d_{f_{i}}}$ for some integer $d_{f_{i}} \geq 2$ for each $i$, and we consider the iterations by $f$ :

$$
X \stackrel{f_{1}}{\longrightarrow} X \stackrel{f_{2}}{\longrightarrow} X \stackrel{f_{3}}{\longrightarrow} \cdots
$$

Let $h_{L}$ be a height function on $X(\bar{K})$ corresponding to $L$. Then we know $c\left(f_{i}\right):=$ $\sup _{x \in X(\bar{K})}\left|\frac{1}{d_{f_{i}}} h_{L}\left(f_{i}(x)\right)-h_{L}(x)\right|<+\infty$ for each $i$. We say that a sequence $f$ is bounded if $c(\boldsymbol{f}):=\sup _{i \geq 1} c\left(f_{i}\right)<+\infty$. Notice that, although we use $h_{L}$ to define boundedness, the property that $\bar{f}$ is bounded is independent of the choice of height functions $h_{L}$ corresponding to $L$. Let $S$ be the shift map which sends $\boldsymbol{f}=\left(f_{i}\right)_{i=1}^{\infty}$ to $S(\boldsymbol{f}):=\left(f_{i+1}\right)_{i=1}^{\infty}$. See $\S 2$ for more details.

After reviewing some basic facts on heights in $\S 1$, we show the following theorem in $\S 2$.

Theorem A (cf. Theorem 2.3). There is a unique way to attach to each bounded sequence $f=\left(f_{i}\right)_{i=1}^{\infty}$ a height function $\widehat{h}_{L, f}: X(\bar{K}) \rightarrow \mathbb{R}$ such that

1991 Mathematics Subject Classification. 11G50, 14G40, 58F23.

Key words and phrases. canonical height, normalized height, random iteration. 
(i) $\sup _{x \in X(\bar{K})}\left|\widehat{h}_{L, \boldsymbol{f}}(x)-h_{L}(x)\right| \leq 2 c(\boldsymbol{f})$;

(ii) $\widehat{h}_{L, S(\boldsymbol{f})} \circ f_{1}=d_{f_{1}} \widehat{h}_{L, \boldsymbol{f}}$.

The function $\widehat{h}_{L, f}$ is independent of the choice of height functions $h_{L}$ corresponding $L$.

Moreover, if $L$ is ample, then $\widehat{h}_{L, f}$ is non-negative, and $\widehat{h}_{L, f}(x)=0$ if and only if $x$ is $\boldsymbol{f}$-preperiodic. (Here a point $x \in X(\bar{K})$ is said to be $\boldsymbol{f}$-preperiodic if the forward orbit of $x$ under $\boldsymbol{f}$, i.e., $O_{\boldsymbol{f}}^{+}(x):=\left\{x, f_{1}(x), f_{2}\left(f_{1}(x)\right), f_{3}\left(f_{2}\left(f_{1}(x)\right)\right), \cdots\right\}$ is finite. $)$

We call $\widehat{h}_{L, \boldsymbol{f}}$ a canonical height function (normalized height function) for $\boldsymbol{f}$.

Note that, for a single morphism $f$, i.e., when $f_{1}=f_{2}=\cdots(=f)$, a canonical height function for $f$ is constructed in [7] and [20]. We remark that some conditions (such as "boundedness") on $\boldsymbol{f}$ are necessary to ensure the existence of height functions that behave well relative to $f$. Indeed, dropping the assumption of boundedness, there is not in general a way to attach to each sequence $\boldsymbol{f}=\left(f_{i}\right)_{i=1}^{\infty}$ a height function $\widehat{h}_{L, \boldsymbol{f}}$ such that (i) $\widehat{h}_{L, \boldsymbol{f}}=$ $h_{L}+O(1)$ and (ii) $\widehat{h}_{L, S(\boldsymbol{f})} \circ f_{1}=d_{f_{1}} \widehat{h}_{L, \boldsymbol{f}}(\mathrm{cf}$. Example 2.5).

Iterations by finitely many morphisms as follows give examples of bounded sequences: Let $g_{1}, \ldots, g_{k}: X \rightarrow X$ be morphisms such that $g_{j}^{*} L \simeq L^{\otimes d_{g_{j}}}$ for some integer $d_{g_{j}} \geq 2$ for $j=1, \ldots, k$. We set $J:=\{1, \ldots, k\}$ and $W:=\prod_{i=1}^{\infty} J$. For $w=\left(w_{i}\right)_{i=1}^{\infty} \in W$, we set $f_{w}=\left(g_{w_{i}}\right)_{i=1}^{\infty}$. Then $\boldsymbol{f}_{w}$ is a bounded sequence. As an immediate consequence of Theorem A, we get the following corollary due to Masseron [13, §2.9].

Corollary B ([13], see also Corollary 2.4(1)(2)). Assume L is ample. Then for any positive integer $D$, the set

$$
\left\{\begin{array}{c|c}
x \in X(\bar{K}) & {[K(x): K] \leq D} \\
x \text { is } \boldsymbol{f}_{w} \text {-preperiodic for some } w \in W
\end{array}\right\}
$$

is finite.

In $\S 3$, noting that averaging of currents over a space with a suitable probability measure and topology is studied in [9], we consider averaging of canonical height functions $\widehat{h}_{L, f_{w}}$ where $w$ runs over $W$. By [12, Theorem 1.2], there is a unique height function $\widehat{h}_{L,\left\{g_{1}, \ldots, g_{k}\right\}}$ on $X(\bar{K})$ with the following two properties: (i) $\widehat{h}_{L,\left\{g_{1}, \ldots, g_{k}\right\}}=h_{L}+O(1)$; and (ii) $\sum_{i=1}^{k} \widehat{h}_{L,\left\{g_{1}, \ldots, g_{k}\right\}} \circ g_{i}=$ $\left(d_{g_{1}}+\cdots+d_{g_{k}}\right) \widehat{h}_{L,\left\{g_{1}, \ldots, g_{k}\right\}}$. Then we have the following proposition.

Proposition $\mathbf{C}$ (cf. Proposition 3.1). We give $J$ the discrete topology, and let $\nu$ be the measure on $J$ that assigns mass $\frac{d_{g_{j}}}{d_{g_{1}+\cdots+g_{g_{k}}}}$ to $j \in J$. Let $\mu:=\prod_{i=1}^{\infty} \nu$ be the product measure on $W$. Then we have, for all $x \in X(\bar{K}), \widehat{h}_{L,\left\{g_{1}, \ldots, g_{k}\right\}}(x)=\int_{W} \widehat{h}_{L, \boldsymbol{f}_{w}}(x) d \mu(w)$.

In $\S 4$, we consider canonical heights of subvarieties of $X_{\bar{K}}$ for $f=\left(f_{i}\right)_{i=1}^{\infty}$, as in [20] for a single morphism $f$. We show in Theorem 4.2 that, assuming some bounded conditions on $f$, there exist a height $\widehat{h}_{L, f}(Y)$ of any subvariety $Y$ of $X_{\bar{K}}$ that behaves well relative to $f$ as in Theorem A. (However, the statement on preperiodicity in Theorem A is changed to the following: If $\left\{Y, f_{1}(Y), f_{2}\left(f_{1}(Y)\right), \cdots\right\}$ is a finite set, then $\widehat{h}_{L, f}(Y)=0$.) In $\S 5$, we give another construction of canonical heights of subvarieties by using adelic sequences. 
In $\S 6$, we consider a local theory in the setting of $[20, \S 2]$. Let $X$ a projective variety over an algebraically closed valuation field $K_{v}$, and $L$ a line bundle on $X$. Let $\|\cdot\|$ be a continuous and bounded metric on $L$. We consider a pair $\bar{f}:=(f, \varphi)$ such that $f: X \rightarrow X$ is a morphism over $K_{v}$ and $\varphi: L^{\otimes d_{f}} \stackrel{\sim}{\rightarrow} f^{*} L$ for some integer $d_{f} \geq 2$. Then we will show in Theorem 6.1 that, assuming some boundedness conditions on $\bar{f}=\left(\overline{f_{i}}\right)_{i=1}^{\infty}$, there exists a bounded and continuous metric $\widehat{\|\cdot\|_{L, \overline{\boldsymbol{f}}}}$ on $L$ satisfying $\varphi_{1}^{*} f_{1}^{*} \widehat{\|\cdot\|}_{L, S(\overline{\boldsymbol{f}})}=\widehat{\|\cdot\|}_{L, \overline{\boldsymbol{f}}}$, where $S$ is the shift map. We call $\widehat{\|\cdot\|}_{L, \bar{f}}$ the admissible metric for $\bar{f}$.

Suppose that $K_{v}=\mathbb{C}, X=\mathbb{P}^{N}$ and $L=\mathcal{O}_{\mathbb{P}^{N}}(1)$ is equipped with the Fubini-Study metric $\|\cdot\|_{F S}$ in the above setting. Let $\mathcal{J}_{\bar{f}}$ be the smallest closed set on $\mathbb{P}^{N}(\mathbb{C})$ such that on its complement the family $\left\{f_{1}, f_{2} \circ f_{1}, f_{3} \circ f_{2} \circ f_{1}, \cdots\right\}$ is normal. As constructed in [9], one has the Green current $T_{\bar{f}}$ on $\mathbb{P}^{N}(\mathbb{C})$, whose support coincides with $\mathcal{J}_{\bar{f}}$ (Proposition 6.3). Then, as noted in $\left[12\right.$, Proposition 3.3.1] for a single morphism $f$, we find $c_{1}\left(\mathcal{O}_{\mathbb{P}^{N}}(1),{\widehat{\|} \cdot \|_{\mathcal{O}^{N}}(1), \bar{f}}\right)=T_{\bar{f}}$, where the left-hand side is the first Chern current of $\mathcal{O}_{\mathbb{P}^{N}}(1)$ with the admissible metric $\widehat{\|\cdot\|}_{\mathcal{O}_{\mathbb{P} N}(1), \bar{f}}$ (Proposition 6.4).

Finally, on $\mathbb{P}^{1}$, we show equidistribution of small points for random iterations as follows. Let $K$ be a number field, and we fix an embedding $\bar{K} \hookrightarrow \mathbb{C}$ so that we regard $\mathbb{P}^{1}(\bar{K})$ as a subset of $\mathbb{P}^{1}(\mathbb{C})$. Let $\bar{f}=\left(\left(f_{i}, \varphi_{i}\right)\right)_{i=1}^{\infty}$ be a sequence of $K$-morphisms on $\mathbb{P}^{1}$ satisfying some boundedness conditions (see $\S 7$ ). Let $\widehat{h}_{\mathcal{O}_{\mathbb{P} 1}(1), f}: \mathbb{P}^{1}(\bar{K}) \rightarrow \mathbb{R}$ be the canonical height function for $f=\left(f_{i}\right)_{i=1}^{\infty}$, and $T_{\bar{f}}$ the Green current for $\bar{f}$ on $\mathbb{P}^{1}(\mathbb{C})$. For a point $x \in \mathbb{P}^{1}(\bar{K})$, we denote the Galois orbit of $x$ by $G(x):=\{g(x) \mid g \in \operatorname{Gal}(\bar{K} / K)\}$. Then we have the following theorem.

Theorem D (cf. Theorem 7.5). Let $\left(x_{j}\right)_{j=1}^{\infty}$ be a sequence of distinct points in $\mathbb{P}^{1}(\bar{K})$ such that $\lim _{j \rightarrow+\infty} \widehat{h}_{\mathcal{O}_{\mathbb{P}}(1), f}\left(x_{j}\right)=0$. Then $\mathrm{w}-\lim _{j \rightarrow+\infty} \frac{1}{\# G\left(x_{j}\right)} \sum_{y \in G\left(x_{j}\right)} \delta_{y}=T_{\bar{f}}$. (Here $\delta_{y}$ denotes the Dirac measure of mass 1 supported in $y$.

In [18], Szpiro-Ullmo-Zhang proved equidistribution of small points on abelian varieties, and ever since much progress has been seen around this subject. On $\mathbb{P}^{1}$, equidistribution of small points will follow from Autissier [1, Proposition 4.1.4 and Remarque in Introduction]. Baker-Hsia [2] explicitly stated and proved equidistribution of small points on $\mathbb{P}^{1}$ for polynomial maps $f$ over global fields satisfying the product formula. See also Chamber-Loir [6], Baker-Rumely [3] and Favre-Rivera-Letelier [8] for equidistribution of small points on $\mathbb{P}^{1}$ over archimedean and non-archimedean fields. Our proof of Theorem D uses Arakelov geometry. Somewhat different ingredients are that we use an estimate of analytic torsions of Bismut-Vasserot [4] and Vojta [19] and that we use only intersections of $C^{\infty}$ hermitian line bundles (i.e., not using $L_{1}^{2}$-intersection theory), in hoping that this might be useful to consider a higher dimensional case (cf. Question 7.2).

Acknowledgment. My deep thanks are due to Professor Laurent Denis. Indeed, this paper is motivated by e-mail from him in October, 2004. He kindly gave me valuable comments and suggestions, and informed me of Dr. Masseron's work [13]. I would also like to thank Professors Atsushi Moriwaki and Joseph Silverman for valuable comments. 


\section{Preliminaries}

In this section, we briefly review some basic facts on heights of closed points and subvarieties.

Let $h_{n v}: \mathbb{P}^{N}(\mathbb{Q}) \rightarrow \mathbb{R}$ be the logarithmic naive height function: For a number field $K$ and $x=\left(x_{0}: \cdots: x_{N}\right) \in \mathbb{P}^{N}(K), h_{n v}(x)$ is given by

$$
h_{n v}(x)=\frac{1}{[K: \mathbb{Q}]}\left[\sum_{P \in \operatorname{Spec}\left(O_{K}\right) \backslash\{0\}} \max _{i}\left\{\log \left\|x_{i}\right\|_{P}\right\}+\sum_{\sigma: K \hookrightarrow \mathbb{C}} \max _{i}\left\{\log \left|\sigma\left(x_{i}\right)\right|\right\}\right],
$$

where $O_{K}$ is the ring of integers of $K$ and $\left\|x_{i}\right\|_{P}=\#\left(O_{K} / P\right)^{-\operatorname{ord}_{P}\left(x_{i}\right)}$.

We will need the following two theorems on height functions. For details of height functions, we refer to [11].

Theorem 1.1 (Weil's height machine, [11] Theorem B.3.2). There is a way to attach to any projective variety $X$ over $\overline{\mathbb{Q}}$ and any line bundle $L$ on $X$ a function

$$
h_{X, L}: X(\overline{\mathbb{Q}}) \rightarrow \mathbb{R}
$$

with the following three properties:

(i) $h_{X, L \otimes M}=h_{X, L}+h_{X, M}+O(1)$ for any line bundles $L$ and $M$ on $X ;$ (Here $O(1)$ denotes a bounded function on $X(\overline{\mathbb{Q}}) ;$ )

(ii) If $X=\mathbb{P}^{N}$ and $L=\mathcal{O}_{\mathbb{P}^{N}}(1)$, then $h_{\mathbb{P}^{N}, \mathcal{O}_{\mathbb{P}^{N}}(1)}=h_{n v}+O(1)$;

(iii) If $f: Y \rightarrow X$ is a morphism of projective varieties and $L$ is a line bundle on $X$, then $h_{Y, f^{*} L}=h_{X, L} \circ f+O(1)$.

Moreover, height functions $h_{X, L}$ are determined up to $O(1)$ by the above three properties.

We say that $h_{X, L}$ is a height function (or a Weil height function) corresponding to $L$. We often write $h_{L}$ for $h_{X, L}$ when $X$ is clear from the context.

Theorem 1.2 ([11] Theorem B.2.3, Theorem B.3.2). Assume $L$ is ample. Let $h_{X, L}$ be $a$ height function corresponding to $L$.

(1) (Northcott's finiteness theorem) For any real number c and positive integer D, the set

is finite.

$$
\left\{x \in X(\overline{\mathbb{Q}}) \mid[\mathbb{Q}(x): \mathbb{Q}] \leq D, h_{X, L}(x) \leq c\right\}
$$

(2) (positivity) There is a constant $c^{\prime}$ such that $h_{X, L}(x) \geq c^{\prime}$ for all $x \in X(\overline{\mathbb{Q}})$.

Next we recall some properties of heights of subvarieties. We refer to [16] for details of arithmetic intersection theory, and to [5] for details of heights of subvarieties.

Let $K$ be a number field, and $O_{K}$ its ring of integers. Let $X$ be a projective variety over $K$, and $L$ a line bundle on $X$. We say that $(\mathcal{X}, \overline{\mathcal{L}})$ is a $C^{\infty}$ model of $(X, L)$ if $\mathcal{X}$ is a projective arithmetic variety over $O_{K}$ (i.e., an integral scheme projective and flat over $\operatorname{Spec}\left(O_{K}\right)$ ) that extends $X$ and if $\overline{\mathcal{L}}$ is a $C^{\infty}$ hermitian $\mathbb{Q}$-line bundle on $\mathcal{X}$ that extends $L$. Here $\overline{\mathcal{L}}$ is called a $C^{\infty}$ hermitian $\mathbb{Q}$-line bundle if $\overline{\mathcal{L}}$ is a pair $\left(\mathcal{L},\left\{\|\cdot\|_{\sigma}\right\}_{\sigma}\right)$ such that $\mathcal{L}$ is a $\mathbb{Q}$-line bundle on $\mathcal{X}$ and $\|\cdot\|_{\sigma}$ 's are $C^{\infty}$ hermitian metrics on $\mathcal{L} \otimes_{K^{\sigma}} \mathbb{C}$ for the embeddings $\sigma: K \hookrightarrow \mathbb{C}$ which are invariant under complex conjugation.

In the rest of this section, we assume $L$ is ample. We fix a $C^{\infty} \operatorname{model}(\mathcal{X}, \overline{\mathcal{L}})$ of $(X, L)$. 
Let $Y$ be a subvariety of $X_{\bar{K}}$. Take a finite extension field $K^{\prime}$ of $K$ such that $Y$ is defined over $K^{\prime}$. Let $O_{K^{\prime}}$ be the ring of integers of $K^{\prime}$, and $p: \mathcal{X} \times_{\operatorname{Spec}\left(O_{K}\right)} \operatorname{Spec}\left(O_{K}^{\prime}\right) \rightarrow \mathcal{X}$ the natural morphism. Let $\mathcal{Y}$ be the Zariski closure of $Y$ in $\mathcal{X} \times_{\operatorname{Spec}\left(O_{K}\right)} \operatorname{Spec}\left(O_{K}^{\prime}\right)$. Then the height of $Y$ with respect to $(\mathcal{X}, \overline{\mathcal{L}})$ is defined by

$$
h_{(\mathcal{X}, \overline{\mathcal{L}})}(Y):=\frac{\widehat{\operatorname{deg}}\left(\widehat{\mathrm{c}}_{1}\left(\left.p^{*} \overline{\mathcal{L}}\right|_{\mathcal{Y}}\right)^{\operatorname{dim} Y+1}\right)}{\left[K^{\prime}: \mathbb{Q}\right](\operatorname{dim} Y+1) \operatorname{deg}\left(\left.L\right|_{Y} ^{\cdot \operatorname{dim} Y}\right)} \quad \in \mathbb{R} .
$$

Theorem $1.3\left([5]\right.$ Proposition 3.2.2). Let $\left(\mathcal{X}_{1}, \overline{\mathcal{L}_{1}}\right)$ and $\left(\mathcal{X}_{2}, \overline{\mathcal{L}_{2}}\right)$ be two $C^{\infty}$ models of $(X, L)$, where we assume $L$ is ample. Then there exists a constant $C$ such that for any subvariety $Y$ of $X_{\bar{K}}$, one has

$$
\left|h_{\left(\mathcal{X}_{1}, \overline{\mathcal{L}_{1}}\right)}(Y)-h_{\left(\mathcal{X}_{2}, \overline{\mathcal{L}_{2}}\right)}(Y)\right| \leq C .
$$

\section{Canonical height Functions}

As in $\S 1$, let $K$ be a number field, $X$ a projective variety over $K$, and $L$ a line bundle on $X$. We fix a height function $h_{L}: X(\bar{K}) \rightarrow \mathbb{R}$ corresponding to $L$.

Let $\mathcal{H}$ be the set of all morphisms $f: X \rightarrow X$ over $K$ such that $f^{*}(L) \simeq L^{\otimes d_{f}}$ for some integer $d_{f} \geq 2$. For $f \in \mathcal{H}$, we set

$$
c(f):=\sup _{x \in X(\bar{K})}\left|\frac{1}{d_{f}} h_{L}(f(x))-h_{L}(x)\right| .
$$

Since $\frac{1}{d_{f}} h_{L} \circ f-h_{L}$ is a bounded function on $X(\bar{K})$ by Theorem 1.1(iii), we see that $c(f)<$ $+\infty$.

Let $f=\left(f_{i}\right)_{i=1}^{\infty}$ be a sequence with $f_{i} \in \mathcal{H}$ for $i \geq 1$. The set of all such sequences is denoted by $\prod_{i=1}^{\infty} \mathcal{H}$. For $f \in \prod_{i=1}^{\infty} \mathcal{H}$, we set

$$
c(\boldsymbol{f}):=\sup _{i \geq 1} c\left(f_{i}\right) \in \mathbb{R} \cup\{+\infty\} .
$$

When $c(f)<+\infty$, we say that $\boldsymbol{f}$ is a bounded sequence.

Suppose $h_{L}^{\prime}$ be another height function corresponding to $L$. Then there is a constant $c$ such that $\sup _{x \in X(\bar{K})}\left|h_{L}(x)-h_{L}^{\prime}(x)\right| \leq c$, and thus

$$
\sup _{x \in X \overline{(K)}}\left|\frac{1}{d_{f}} h_{L}^{\prime}(f(x))-h_{L}^{\prime}(x)\right| \leq \sup _{x \in X \bar{K})}\left|\frac{1}{d_{f}} h_{L}(f(x))-h_{L}(x)\right|+\left(\frac{1}{d_{f}}+1\right) c \leq c(f)+2 c .
$$

This shows that the property that $f$ is bounded is independent of the choice of height functions $h_{L}$ corresponding to $L$.

Let $\mathcal{B}$ be the set of all bounded sequences in $\prod_{i=1}^{\infty} \mathcal{H}$. For any nonnegative number $c$, we define the subset $\mathcal{B}_{c}$ of $\mathcal{B}$ by

$$
\mathcal{B}_{c}:=\left\{\boldsymbol{f}=\left(f_{i}\right)_{i=1}^{\infty} \in \mathcal{B} \mid c(\boldsymbol{f}) \leq c\right\} .
$$

Let $S: \prod_{i=1}^{\infty} \mathcal{H} \rightarrow \prod_{i=1}^{\infty} \mathcal{H}$ be the shift map which sends $\boldsymbol{f}=\left(f_{i}\right)_{i=1}^{\infty} \in \prod_{i=1}^{\infty} \mathcal{H}$ to $S(\boldsymbol{f}):=$ $\left(f_{i+1}\right)_{i=1}^{\infty} \in \prod_{i=1}^{\infty} \mathcal{H}$. Since $c(S(\boldsymbol{f})) \leq c(\boldsymbol{f}), S$ maps $\mathcal{B}$ into $\mathcal{B}$, and $\mathcal{B}_{c}$ into $\mathcal{B}_{c}$ for any $c$.

For $\boldsymbol{f}=\left(f_{i}\right)_{i=1}^{\infty} \in \prod_{i=1}^{\infty} \mathcal{H}$ and $x \in X(\bar{K})$, we consider iterated points by $\boldsymbol{f}$ with the initial point $x$ :

$$
x \mapsto f_{1}(x) \mapsto f_{2}\left(f_{1}(x)\right) \mapsto f_{3}\left(f_{2}\left(f_{1}(x)\right)\right) \mapsto \cdots .
$$


The set $\left\{x, f_{1}(x), f_{2}\left(f_{1}(x)\right), f_{3}\left(f_{2}\left(f_{1}(x)\right)\right), \cdots\right\}$ is called the forward orbit of $x$ under $\boldsymbol{f}$, and is denoted by $O_{\boldsymbol{f}}^{+}(x)$. A point $x \in X(\bar{K})$ is said to be $\boldsymbol{f}$-preperiodic if $O_{\boldsymbol{f}}^{+}(x)$ is a finite set. Note that, when $f_{1}=f_{2}=\cdots(=f)$, the forward orbit under $\boldsymbol{f}$ is just the forward orbit under $f$ in the usual sense, and an $f$-preperiodic point is just a usual $f$-preperiodic point.

Example 2.1. We consider iterations by a finite number of morphisms $g_{1}, \ldots, g_{k} \in \mathcal{H}$. We set $J:=\{1, \ldots, k\}$ and $W:=\prod_{i=1}^{\infty} J$. For $w=\left(w_{i}\right)_{i=1}^{\infty} \in W$, we set $\boldsymbol{f}_{w}=\left(g_{w_{i}}\right)_{i=1}^{\infty}$. Put $c:=\max \left\{c\left(g_{1}\right), \ldots, c\left(g_{k}\right)\right\}$. Then $\left\{f_{w} \mid w \in W\right\} \subset \mathcal{B}_{c}$.

Example 2.2. We give some concrete examples. Let $K=\mathbb{Q}, X=\mathbb{P}^{N}$ and $L=\mathcal{O}_{\mathbb{P}^{N}}(1)$. For a fixed height function, we take the naive height function $h_{n v}: \mathbb{P}^{N}(\overline{\mathbb{Q}}) \rightarrow \mathbb{R}$.

(1) For each $m \geq 2$, let $g_{m}^{\prime}: \mathbb{P}^{N} \rightarrow \mathbb{P}^{N}$ be the morphism of degree $d_{g_{m}^{\prime}}=m$ defined by

$$
g_{m}^{\prime}\left(x_{0}: \cdots: x_{N}\right)=\left(x_{0}^{m}: \cdots: x_{N}^{m}\right) .
$$

Then $c\left(g_{m}^{\prime}\right)=0$. Thus any sequence $\boldsymbol{f}=\left(f_{i}\right)_{i=1}^{\infty}$ such that, for each $i$, there is $m_{i}$ with $f_{i}=g_{m_{i}}^{\prime}$ belongs to $\mathcal{B}_{0}$.

(2) For each $m \geq 2$, let $g_{m}^{\prime \prime}: \mathbb{P}^{N} \rightarrow \mathbb{P}^{N}$ be the morphism of degree $d_{g_{m}^{\prime \prime}}=m$ defined by

$$
g_{m}^{\prime \prime}\left(x_{0}: x_{1}: \cdots: x_{N}\right)=\left(x_{0}^{m}+x_{1}^{m}: x_{1}^{m}: \cdots: x_{N}^{m}\right) .
$$

Then it is easy to see $c\left(g_{m}^{\prime \prime}\right) \leq \log 2$. Thus any sequence $f=\left(f_{i}\right)_{i=1}^{\infty}$ such that, for each $i$, there is $m_{i}$ with $f_{i}=g_{m_{i}}^{\prime \prime}$ belongs to $\mathcal{B}_{\log 2}$.

Theorem 2.3. Let $X$ be a projective variety over a number field $K$, and $L$ a line bundle on $X$. Let $h_{L}: X(\bar{K}) \rightarrow \mathbb{R}$ be a height function corresponding to $L$.

(1) There is a unique way to attach to each bounded sequence $\boldsymbol{f}=\left(f_{i}\right)_{i=1}^{\infty} \in \mathcal{B}$ a height function

$$
\widehat{h}_{L, f}: X(\bar{K}) \rightarrow \mathbb{R}
$$

such that

(i) $\sup _{x \in X(\bar{K})}\left|\widehat{h}_{L, \boldsymbol{f}}(x)-h_{L}(x)\right| \leq 2 c(\boldsymbol{f})$;

(ii) $\widehat{h}_{L, S(\boldsymbol{f})} \circ f_{1}=d_{f_{1}} \widehat{h}_{L, \boldsymbol{f}}$.

Moreover, $\widehat{h}_{L, f}$ is independent of the choice of height functions $h_{L}$ corresponding to $L$.

(2) Assume $L$ is ample. Then $\widehat{h}_{L, f}$ satisfies the following properties:

(iii) $\widehat{h}_{L, f}(x) \geq 0$ for all $x \in X(\bar{K})$;

(iv) $\widehat{h}_{L, \boldsymbol{f}}(x)=0$ if and only if $x$ is $\boldsymbol{f}$-preperiodic.

We call $\widehat{h}_{L, \boldsymbol{f}}$ a canonical height function (normalized height function) for $\boldsymbol{f}$.

Proof. (1) We first construct $\widehat{h}_{L, \boldsymbol{f}}$ for $\boldsymbol{f} \in \mathcal{B}$. By the definition of $c(\boldsymbol{f})$, we have for any $i$

$$
\sup _{x \in X(\bar{K})}\left|\frac{1}{d_{f_{i}}} h_{L}\left(f_{i}(x)\right)-h_{L}(x)\right| \leq c(\boldsymbol{f}) \quad(<+\infty) .
$$

For $i=0$ we set $h_{0}:=h_{L}$, and for $i \geq 1$ we set

$$
h_{i}:=\frac{1}{\prod_{\alpha=1}^{i} d_{f_{\alpha}}} h_{L} \circ f_{i} \circ f_{i-1} \circ \cdots \circ f_{1} .
$$


Claim 2.3.1. For $x \in X(\bar{K}),\left\{h_{i}(x)\right\}_{i=0}^{\infty}$ is a Cauchy sequence.

Indeed, we have

$$
\begin{aligned}
\left|h_{i+1}(x)-h_{i}(x)\right| & =\left|\frac{1}{\prod_{\alpha=1}^{i+1} d_{f_{\alpha}}} h_{L}\left(f_{i+1} \circ \cdots \circ f_{1}(x)\right)-\frac{1}{\prod_{\alpha=1}^{i} d_{f_{\alpha}}} h_{L}\left(f_{i} \circ \cdots \circ f_{1}(x)\right)\right| \\
& =\frac{1}{\prod_{\alpha=1}^{i} d_{f_{\alpha}}}\left|\frac{1}{d_{f_{i+1}}} h_{L}\left(f_{i+1}\left(f_{i} \circ \cdots \circ f_{1}(x)\right)\right)-h_{L}\left(f_{i} \circ \cdots \circ f_{1}(x)\right)\right| \\
& \leq \frac{c(\boldsymbol{f})}{\prod_{\alpha=1}^{i} d_{f_{\alpha}}} \leq \frac{c(\boldsymbol{f})}{2^{i}} .
\end{aligned}
$$

Thus we get the claim. We define $\widehat{h}_{L, f}$ to be

$$
\widehat{h}_{L, f}(x):=\lim _{i \rightarrow \infty} h_{i}(x)=\lim _{i \rightarrow \infty} \frac{1}{\prod_{\alpha=1}^{i} d_{f_{\alpha}}} h_{L}\left(f_{i} \circ \cdots \circ f_{1}(x)\right) .
$$

Let us check that $\widehat{h}_{L, f}(x)$ satisfies the conditions (i) and (ii). Since

$$
\left|h_{i}(x)-h_{L}(x)\right| \leq \sum_{\alpha=0}^{i-1}\left|h_{\alpha+1}(x)-h_{\alpha}(x)\right| \leq \sum_{\alpha=0}^{i-1} \frac{c(\boldsymbol{f})}{2^{\alpha}} \leq 2 c(\boldsymbol{f}),
$$

we obtain (i) by letting $i$ to the infinity.

Since $\widehat{h}_{L, S(\boldsymbol{f})}(y)=\lim _{i \rightarrow \infty} \frac{1}{\prod_{\alpha=1}^{i} d_{f_{\alpha+1}}} h_{L}\left(f_{i+1} \circ f_{i} \circ \cdots \circ f_{2}(y)\right)$, substituting $y=f_{1}(x)$ gives

$$
\begin{aligned}
\widehat{h}_{L, S(\boldsymbol{f})}\left(f_{1}(x)\right) & =\lim _{i \rightarrow \infty} \frac{1}{\prod_{\alpha=1}^{i} d_{f_{\alpha+1}}} h_{L}\left(f_{i+1} \circ f_{i} \circ \cdots \circ f_{2} \circ f_{1}(x)\right) \\
& =d_{f_{1}} \lim _{i \rightarrow \infty} \frac{1}{\prod_{\alpha=1}^{i+1} d_{f_{\alpha}}} h_{L}\left(f_{i+1} \circ f_{i} \circ \cdots \circ f_{2} \circ f_{1}(x)\right)=d_{f_{1}} \widehat{h}_{L, \boldsymbol{f}}(x) .
\end{aligned}
$$

Thus we get (ii).

To show the uniqueness of $\left\{\widehat{h}_{L, \boldsymbol{f}}\right\}_{\boldsymbol{f} \in \mathcal{B}}$, suppose $\left\{\widehat{h}_{L, \boldsymbol{f}}^{\prime}\right\}_{\boldsymbol{f} \in \mathcal{B}}$ are functions satisfying (i) and (ii). By (ii), we have

$$
\begin{aligned}
\sup _{x \in X(\bar{K})}\left|\widehat{h}_{L, \boldsymbol{f}}^{\prime}(x)-\widehat{h}_{L, \boldsymbol{f}}(x)\right| & \leq \frac{1}{d_{f_{1}}} \sup _{x \in X(\bar{K})}\left|\widehat{h}_{L, S(\boldsymbol{f})}^{\prime}(x)-\widehat{h}_{L, S(\boldsymbol{f})}(x)\right| \\
& \leq \cdots \leq \frac{1}{\prod_{\alpha=1}^{i} d_{f_{\alpha}}} \sup _{x \in X(\bar{K})}\left|\widehat{h}_{L, S^{i}(\boldsymbol{f})}^{\prime}(x)-\widehat{h}_{L, S^{i}(\boldsymbol{f})}(x)\right| \leq \frac{4 c\left(S^{i}(\boldsymbol{f})\right)}{\prod_{\alpha=1}^{i} d_{f_{\alpha}}} .
\end{aligned}
$$

Since $c\left(S^{i}(\boldsymbol{f})\right) \leq c(\boldsymbol{f})$, we obtain $\sup _{x \in X(\bar{K})}\left|\widehat{h}_{L, \boldsymbol{f}}^{\prime}(x)-\widehat{h}_{L, \boldsymbol{f}}(x)\right|=0$ by letting $i$ to the infinity, whence $\widehat{h}_{L, f}=\widehat{h}_{L, f}^{\prime}$.

Since the difference of two height functions corresponding to $L$ is bounded on $X(\bar{K})$, it follows from (2.1) that $\widehat{h}_{L, f}$ is independent of the choice of height functions $h_{L}$.

(2) Now assuming $L$ is ample, let us see (iii) and (iv). Since $h_{L}$ is bounded below by Theorem 1.2(2), we get (iii) by (2.1). 
Next we show (iv). Suppose $\widehat{h}_{L, \boldsymbol{f}}(x)=0$. Take a finite extension field $K^{\prime}$ of $K$ such that $x$ is defined over $K^{\prime}$. Then for any $i \geq 1, f_{i} \circ \cdots \circ f_{1}(x)$ is also defined over $K^{\prime}$. We set

$$
T:=\left\{y \in X\left(K^{\prime}\right) \mid h_{L}(y) \leq 2 c(\boldsymbol{f})\right\}
$$

We claim that the forward orbit $O_{\boldsymbol{f}}^{+}(x)$ is contained in $T$. Indeed, since $\widehat{h}_{L, \boldsymbol{f}}(x)=0$ and $\left|\widehat{h}_{L, \boldsymbol{f}}(x)-h_{L}(x)\right| \leq 2 c(\boldsymbol{f})$, we see $x \in T$. For $i \geq 1$, we have

$$
\widehat{h}_{L, S^{i}(\boldsymbol{f})}\left(f_{i} \circ \cdots \circ f_{1}(x)\right)=\left(\prod_{\alpha=1}^{i} d_{f_{\alpha}}\right) \widehat{h}_{L, \boldsymbol{f}}(x)=0 .
$$

It follows from

$$
\left|\widehat{h}_{L, S^{i}(\boldsymbol{f})}\left(f_{i} \circ \cdots \circ f_{1}(x)\right)-h_{L}\left(f_{i} \circ \cdots \circ f_{1}(x)\right)\right| \leq 2 c\left(S^{i}(\boldsymbol{f})\right) \leq 2 c(\boldsymbol{f})
$$

that $h_{L}\left(f_{i} \circ \cdots \circ f_{1}(x)\right) \leq 2 c(\boldsymbol{f})$. Thus we get the claim. Since $T$ is a finite set by Northcott's finiteness theorem (Theorem 1.2), so is $O_{f}^{+}(x)$. In other words, $x$ is $f$-preperiodic.

Finally we will show that $x$ is $\boldsymbol{f}$-preperiodic then $\widehat{h}_{L, \boldsymbol{f}}(x)=0$. To see this, suppose $\widehat{h}_{L, \boldsymbol{f}}(x)=a>0$. Then

$$
\widehat{h}_{L, S^{i}(\boldsymbol{f})}\left(f_{i} \circ \cdots \circ f_{1}(x)\right)=\left(\prod_{\alpha=1}^{i} d_{f_{\alpha}}\right) a \rightarrow+\infty
$$

as $i$ tends to the infinity. Thus by (2.3), $O_{\boldsymbol{f}}^{+}(x)$ cannot be finite.

The following corollary is an immediate consequence of Theorem 2.3. We remark that Corollary $2.4(2)$ is due to Masseron $[13, \S 2.9]$.

Corollary 2.4. Assume $L$ is ample.

(1) Let $c$ be a nonnegative number, and $D$ a positive integer. Then the set

$$
\bigcup_{\boldsymbol{f} \in \mathcal{B}_{c}}\{x \in X(\bar{K}) \mid[K(x): K] \leq D, x \text { is } \boldsymbol{f} \text {-preperiodic }\}
$$

is finite.

(2) Let $g_{1}, \ldots, g_{k}$ be elements of $\mathcal{H}$. As in Example 2.1, we set $W:=\prod_{i=1}^{\infty}\{1, \ldots, k\}$, and $\boldsymbol{f}_{w}=\left(g_{w_{i}}\right)_{i=1}^{\infty}$ for $w=\left(w_{i}\right)_{i=1}^{\infty} \in W$. Then for any positive integer $D$, the set

$$
\left\{\begin{array}{c|c}
x \in X(\bar{K}) \mid \begin{array}{c}
{[K(x): K] \leq D,} \\
x \text { is } \boldsymbol{f}_{w} \text {-preperiodic for some } w \in W
\end{array}
\end{array}\right\}
$$

is finite.

Proof. By Theorem 2.3, we obtain for $\boldsymbol{f} \in \mathcal{B}_{c}$,

$$
\left\{\begin{array}{l|l}
x \in X(\bar{K}) & \begin{array}{c}
{[K(x): K] \leq D,} \\
x \text { is } f \text {-preperiodic }
\end{array}
\end{array}\right\}=\left\{\begin{array}{l|l}
x \in X(\bar{K}) & \begin{array}{c}
{[K(x): K] \leq D,} \\
\widehat{h}_{L, \boldsymbol{f}}(x)=0
\end{array}
\end{array}\right\} .
$$

Since $\sup _{x \in X(\bar{K})}\left|\widehat{h}_{L, \boldsymbol{f}}(x)-h_{L}(x)\right| \leq 2 c(\boldsymbol{f}) \leq 2 c$, we find that the above set is a subset of

$$
\left\{x \in X(\bar{K}) \mid[K(x): K] \leq D, h_{L}(x) \leq 2 c\right\} .
$$


This set is finite by Northcott's finiteness theorem (Theorem 1.2), and is independent of $f \in \mathcal{B}_{c}$. Thus we get the assertion (1). Since $\left\{\boldsymbol{f}_{w} \mid w \in W\right\}$ is contained in $\mathcal{B}_{c}$ with $c:=\max \left\{c\left(g_{1}\right), \ldots, c\left(g_{k}\right)\right\}$, the assertion (2) follows from (1).

Example 2.5. By dropping the assumption of boundedness in Theorem 2.3, one can ask the following question: Is there a way to attach to each sequence $f \in \prod_{i=1}^{\infty} \mathcal{H}$ a height function $\widehat{h}_{L, f}: X(\bar{K}) \rightarrow \mathbb{R}$ such that (i) $\widehat{h}_{L, f}=h_{L}+O(1)$ and (ii) $\widehat{h}_{L, S(\boldsymbol{f})} \circ f_{1}=d_{f_{1}} \widehat{h}_{L, \boldsymbol{f}}$ ?

The following example shows that this is not possible in general, and thus some conditions (such as "boundedness") on $f$ are necessary to ensure the existence of height functions that behave well relative to $f$.

We define $F_{i}$ inductively:

$$
\begin{aligned}
F_{1}(x) & =x(x-1) \\
F_{i}(x) & =x\left(x-F_{i-1} \circ \cdots \circ F_{1}(i)\right) \quad(i \geq 2) .
\end{aligned}
$$

We define $f_{i}: \mathbb{P}^{1} \rightarrow \mathbb{P}^{1}$ by

$$
f_{i}\left(\left(x_{0}: x_{1}\right)\right)=\left(x_{0}^{2}: F_{i}\left(\frac{x_{1}}{x_{0}}\right) x_{0}^{2}\right) .
$$

Then $f_{i}$ is defined over $\mathbb{Q}$ and $d_{f_{i}}=2$ for any $i \geq 1$. We put $p_{i}=(1: i) \in \mathbb{P}^{1}(\mathbb{Q})$ for $i \geq 0$. Then $f_{\alpha}\left(p_{0}\right)=p_{0}$ for any $\alpha \geq 1$, and $f_{i} \circ f_{i-1} \circ \cdots \circ f_{1}\left(p_{i}\right)=p_{0}$.

Suppose there exists a height function $\widehat{h}_{\mathcal{O}_{\mathbb{P} 1}(1), \boldsymbol{f}}: \mathbb{P}^{1}(\overline{\mathbb{Q}}) \rightarrow \mathbb{R}$ satisfying (i) $\widehat{h}_{\mathcal{O}_{\mathbb{P} 1}(1), \boldsymbol{f}}=$ $h_{n v}+O(1)$ and (ii) $\widehat{h}_{\mathcal{O}_{\mathbb{P}}^{1}(1), S^{i}(\boldsymbol{f})} \circ f_{1}=2 \widehat{h}_{\mathcal{O}_{\mathbb{P} 1}(1), S^{i-1}(\boldsymbol{f})}$ for $i \geq 1$. Then by (ii),

$$
\begin{aligned}
\widehat{h}_{\mathcal{O}_{\mathbb{P}}(1), \boldsymbol{f}}\left(p_{i}\right) & =\frac{1}{2^{i}} \widehat{h}_{\mathcal{O}_{\mathbb{P}}(1), S^{i}(\boldsymbol{f})}\left(f_{i} \circ f_{i-1} \circ \cdots \circ f_{1}\left(p_{i}\right)\right) \\
& =\frac{1}{2^{i}} \widehat{h}_{\mathcal{O}_{\mathbb{P}}(1), S^{i}(\boldsymbol{f})}\left(p_{0}\right)=\frac{1}{2^{i}} \widehat{h}_{\mathcal{O}_{\mathbb{P}}(1), S^{i}(\boldsymbol{f})}\left(f_{i} \circ f_{i-1} \circ \cdots \circ f_{1}\left(p_{0}\right)\right)=\widehat{h}_{\mathcal{O}_{\mathbb{P}^{1}}(1), \boldsymbol{f}}\left(p_{0}\right)
\end{aligned}
$$

for any $i \geq 1$. Thus the set $T:=\left\{x \in \mathbb{P}^{1}(\mathbb{Q}) \mid \widehat{h}_{\mathcal{O}_{\mathbb{P} 1}(1), f}(x)=\widehat{h}_{\mathcal{O}_{\mathbb{P} 1}(1), f}\left(p_{0}\right)\right\}$ is an infinite set. This contradicts $(i)$, as $\widehat{h}_{\mathcal{O}_{\mathbb{P}}(1), f}$ does not satisfy Northcott's finiteness property.

We also give two remarks on possible extentions of Theorem 2.3.

Remark 2.6. We replace an (integral) line bundle $L$ by an $\mathbb{R}$-line bundle, and integers $d_{f_{i}}$ by real numbers. We fix a positive real number $\varepsilon>0$. Let $X$ be a projective variety over a number field $K$, and $L$ an $\mathbb{R}$-line bundle on $X$. We consider sequences $\boldsymbol{f}=\left(f_{i}\right)_{i=1}^{\infty}$, where $f_{i}: X \rightarrow X$ is a morphism such that $f_{i}^{*}(L) \simeq L^{\otimes d_{f_{i}}}$ for some real number $d_{f_{i}} \geq(1+\varepsilon)$. Then, with the same definition of boundedness, the corresponding statements to Theorem 2.3 hold. Note that the property of Theorem 2.3 (1)(i) is replaced by

$$
\text { (i)' } \sup _{x \in X(\bar{K})}\left|\widehat{h}_{L, \boldsymbol{f}}(x)-h_{L}(x)\right| \leq\left(1+\frac{1}{\varepsilon}\right) c(\boldsymbol{f}) \text {. }
$$

Remark 2.7. We replace a number field $K$ by $\overline{\mathbb{Q}}$. Let $X$ be a projective variety over $\overline{\mathbb{Q}}$, and $L$ a line bundle on $X$. We consider sequences $f=\left(f_{i}\right)_{i=1}^{\infty}$, where $f_{i}: X \rightarrow X$ is a morphism over $\overline{\mathbb{Q}}$ such that $f_{i}^{*}(L) \simeq L^{\otimes d_{f_{i}}}$ for some integer $d_{f_{i}} \geq 2$. Then, with the same definition of boundedness, the corresponding statements to Theorem 2.3(1) and (2)(i) hold. However, the corresponding statement to Theorem 2.3(2)(ii) does not hold, and must be replaced by 
(iv)' $\widehat{h}_{L, \boldsymbol{f}}(x)=0$ if $x$ is $\boldsymbol{f}$-preperiodic.

To see that the "only if" part of (iv)' fails in general, we give an example as follows. Let $p_{1}, p_{2}, \ldots$ be distinct prime numbers. Let $\zeta_{i}$ be a $p_{i}$-th primitive root of unity for each $i$. We set $X=\mathbb{P}_{\mathbb{Q}} \frac{1}{\text { and }} L=\mathcal{O}_{\mathbb{P} \frac{1}{\mathbb{Q}}}(1)$, and consider the logarithmic naive height function $h_{n v}$. We define $f_{i}: \mathbb{P}_{\overline{\mathbb{Q}}}^{1} \rightarrow \mathbb{P}_{\mathbb{Q}}^{1}$ by $f_{i}\left(\left(x_{0}: x_{1}\right)\right)=\left(\zeta_{i} x_{0}^{2}: x_{1}^{2}\right)$. Then

$$
h_{n v}\left(f_{i}\left(\left(x_{0}: x_{1}\right)\right)\right)=h_{n v}\left(\left(\zeta_{i} x_{0}^{2}: x_{1}^{2}\right)\right)=2 h_{n v}\left(\left(x_{0}: x_{1}\right)\right) .
$$

This shows that $\boldsymbol{f}=\left(f_{i}\right)_{i=1}^{\infty}$ is bounded, and $\widehat{h}_{L, \boldsymbol{f}}=h_{n v}$. Now we take $x_{0}=(1: 1)$. On one hand, $\widehat{h}_{L, f}\left(x_{0}\right)=h_{n v}\left(x_{0}\right)=0$. On the other hand, since $f_{i} \circ \cdots \circ f_{1}\left(x_{0}\right)=\left(\zeta_{i} \zeta_{i-1}^{2} \cdots \zeta_{1}^{2^{i}}: 1\right)$, $O_{\boldsymbol{f}}^{+}\left(x_{0}\right)$ is an infinite set. Thus the "only if" part of (iv)' fails in this example.

\section{Averaging of CANONICAL HeIGHT FUNCTIONS}

Let $X$ be a projective variety over a number field $K$, and $L$ a line bundle on $X$. As in Example 2.1, let $g_{1}, \ldots, g_{k}$ be elements of $\mathcal{H}$, and we set $J:=\{1, \ldots, k\}, W:=\prod_{i=1}^{\infty} J$, and $\boldsymbol{f}_{w}=\left(g_{w_{i}}\right)_{i=1}^{\infty}$ for $w=\left(w_{i}\right)_{i=1}^{\infty} \in W$. Let $\widehat{h}_{L, \boldsymbol{f}_{w}}$ be the canonical height function for $\boldsymbol{f}_{w}$.

In this section, noting that averaging of currents over a space with a suitable probability measure and topology is studied in [9], we would like to consider averaging of $\widehat{h}_{L, \boldsymbol{f}_{w}}$ over $W$.

First we recall [12, Theorem 1.2]. Since $g_{j}^{*} L \simeq L^{\otimes d_{g_{j}}}$, we have $g_{1}^{*} L \otimes \cdots \otimes g_{k}^{*} L \simeq$ $L^{\otimes\left(d_{g_{1}}+\cdots+d_{g_{k}}\right)}$. Thus $\left(X ; g_{1}, \ldots, g_{k}\right)$ becomes a particular case of what we call a dynamical eigensystem for $L$ of degree $d_{g_{1}}+\cdots+d_{g_{k}}$. Then we have the canonical height function

$$
\widehat{h}_{L,\left\{g_{1}, \ldots, g_{k}\right\}}: X(\bar{K}) \rightarrow \mathbb{R}
$$

for $\left(X ; g_{1}, \ldots, g_{k}\right)$ characterized by the following two properties: (i) $\widehat{h}_{L,\left\{g_{1}, \ldots, g_{k}\right\}}=h_{L}+O(1)$; and (ii) $\sum_{j=1}^{k} \widehat{h}_{L,\left\{g_{1}, \ldots, g_{k}\right\}} \circ g_{j}=\left(d_{g_{1}}+\cdots+d_{g_{k}}\right) \widehat{h}_{L,\left\{g_{1}, \ldots, g_{k}\right\}}$.

Proposition 3.1. We give $J$ the discrete topology, and let $\nu$ be the measure on $J$ that assigns mass $\frac{d_{g_{j}}}{d_{g_{1}}+\cdots+d_{g_{k}}}$ to $j \in J$. Let $\mu:=\prod_{i=1}^{\infty} \nu$ be the product measure on $W$. Then we have, for all $x \in X(\bar{K})$,

$$
\widehat{h}_{L,\left\{g_{1}, \ldots, g_{k}\right\}}(x)=\int_{W} \widehat{h}_{f_{w}}(x) d \mu(w) .
$$

Proof. As in Example 2.1, we set $c:=\max \left\{c\left(g_{1}\right), \ldots, c\left(g_{k}\right)\right\}$. For $w=\left(w_{i}\right)_{i=1}^{\infty} \in W$ and $x \in X(\bar{K})$, we put

$$
h_{i}(x, w):=\frac{1}{\prod_{\alpha=1}^{i} d_{g_{w_{\alpha}}}} h_{L}\left(g_{w_{i}} \circ \cdots \circ g_{w_{1}}(x)\right) .
$$

By (2.1), $\lim _{i \rightarrow \infty} h_{i}(x, w)=\widehat{h}_{L, \boldsymbol{f}_{w}}(x)$. By $(2.2),\left|h_{i}(x, w)\right| \leq 2 c+\left|h_{L}(x)\right|$. Then, if we fix $x,\left\{h_{i}(x, w)\right\}_{i=1}^{\infty}$ is a sequence of integral functions on $W$ bounded by $2 c+\left|h_{L}(x)\right|$. The Lebesgue convergence theorem implies

$$
\int_{W} \widehat{h}_{L, \boldsymbol{f}_{w}}(x) d \mu(w)=\lim _{i \rightarrow \infty} \int_{W} h_{i}(x, w) d \mu(w) .
$$

We set $\widehat{h}^{\prime}(x):=\int_{W} \widehat{h}_{L, \boldsymbol{f}_{w}}(x) d \mu(w)$. We will show that as a function on $X(\bar{K}), \widehat{h}^{\prime}$ satisfies the properties (i) and (ii) of $\widehat{h}_{L,\left\{g_{1}, \ldots, g_{k}\right\}}$ 
Since

$$
\left|\widehat{h}^{\prime}(x)-h_{L}(x)\right| \leq\left|\int_{W}\left(\widehat{h}_{L, \boldsymbol{f}_{w}}(x)-h_{L}(x)\right) d \mu(w)\right| \leq 2 c,
$$

we get (i). To show (ii), we note the following equality:

$$
\begin{aligned}
\int_{W} & h_{i}(x, w) d \mu(w)=\int_{W} \frac{h_{L}\left(g_{w_{i}} \circ \cdots \circ g_{w_{1}}(x)\right)}{\prod_{\alpha=1}^{i} d_{g_{w_{\alpha}}}} d \mu(w) \\
= & \sum_{w_{1}, \ldots, w_{i}=1}^{k} \frac{h_{L}\left(g_{w_{i}} \circ \cdots \circ g_{w_{1}}(x)\right)}{\prod_{\alpha=1}^{i} d_{g_{w_{\alpha}}}} \prod_{\alpha=1}^{i} \frac{d_{g_{w_{\alpha}}}}{d_{g_{1}}+\cdots+d_{g_{k}}}=\sum_{w_{1}, \ldots, w_{i}=1}^{k} \frac{h_{L}\left(g_{w_{i}} \circ \cdots \circ g_{w_{1}}(x)\right)}{\left(d_{g_{1}}+\cdots+d_{g_{k}}\right)^{i}} .
\end{aligned}
$$

Then we have

$$
\begin{aligned}
\sum_{j=1}^{k} \widehat{h}^{\prime}\left(g_{j}(x)\right) & =\lim _{i \rightarrow \infty} \sum_{j=1}^{k} \int_{W} h_{i}\left(g_{j}(x), w\right) d \mu(w) \\
& =\lim _{i \rightarrow \infty} \sum_{j=1}^{k} \sum_{w_{1}, \ldots, w_{i}=1}^{k} \frac{h_{L}\left(g_{w_{i}} \circ \cdots \circ g_{w_{1}}\left(g_{j}(x)\right)\right)}{\left(d_{g_{1}}+\cdots+d_{g_{k}}\right)^{i}} \\
& =\lim _{i \rightarrow \infty}\left(d_{g_{1}}+\cdots+d_{g_{k}}\right) \int_{W} h_{i+1}(x, w) d \mu(w)=\left(d_{g_{1}}+\cdots+d_{g_{k}}\right) \widehat{h}^{\prime}(x) .
\end{aligned}
$$

Thus we get (ii). By the uniqueness of $\widehat{h}_{L,\left\{g_{1}, \ldots, g_{k}\right\}}$, we get the assertion.

Remark 3.2. As in [9, Remark 1], it is possible to consider other index sets $J$ with suitable probability measures and topologies.

\section{Canonical heights of subvarieties, I}

As in $\S 1$, let $K$ be a number field, $O_{K}$ its ring of integers, $X$ a projective variety over $K$, and $L$ a line bundle on $X$. We assume $L$ is ample. We fix a $C^{\infty}$ model $(\mathcal{X}, \overline{\mathcal{L}})$ of $(X, L)$.

Let $f$ be an element of $\mathcal{H}$, that is, $f: X \rightarrow X$ is a morphism over $K$ such that $f^{*}(L) \simeq L^{\otimes d_{f}}$ for some integer $d_{f} \geq 2$. Since we assume $L$ is ample, $f$ is finite.

Lemma 4.1. There exists a constant $\widetilde{c}$ such that, for any subvariety $Y$ of $X_{\bar{K}}$, one has

$$
\left|\frac{1}{d_{f}} h_{(\mathcal{X}, \overline{\mathcal{L}})}(f(Y))-h_{(\mathcal{X}, \overline{\mathcal{L}})}(Y)\right| \leq \widetilde{c} .
$$

Proof. Let $\mathcal{X}_{1}$ be the normalization of the morphism $X \stackrel{f}{\longrightarrow} X \hookrightarrow \mathcal{X}$, and $\tilde{f}: \mathcal{X}_{1} \rightarrow \mathcal{X}$ the induced morphism. Then $\left(\mathcal{X}_{1}, \tilde{f}^{*}(\overline{\mathcal{L}})\right)$ is a $C^{\infty}$ model of $\left(X, L^{\otimes d_{f}}\right)$. By Theorem 1.3, there exists a constant $\tilde{c}^{\prime}$ such that, for any subvariety $Y$ of $X_{\bar{K}}$,

$$
\left|h_{\left(\mathcal{X}_{1}, \tilde{f}^{*}(\overline{\mathcal{L}})\right)}(Y)-h_{\left(\mathcal{X}, \overline{\mathcal{L}}^{\otimes d_{f}}\right)}(Y)\right| \leq \tilde{c}^{\prime} .
$$

On the other hand, by the projection formula, we have $h_{\left(\mathcal{X}_{1}, \tilde{f}^{*}(\overline{\mathcal{L}})\right)}(Y)=h_{(\mathcal{X}, \overline{\mathcal{L}})}(f(Y))$. Since $h_{\left(\mathcal{X}, \overline{\mathcal{L}}^{\left.\otimes d_{f}\right)}\right.}(Y)=d_{f} h_{(\mathcal{X}, \overline{\mathcal{L}})}(Y)$, we obtain the assertion with $\widetilde{c}=\frac{\tilde{c}^{\prime}}{d_{f}}$. 
For $f \in \mathcal{H}$, we set

$$
\widetilde{c}(f):=\sup _{Y \subset X_{\bar{K}}}\left|\frac{1}{d_{f}} h_{(\mathcal{X}, \overline{\mathcal{L}})}(f(Y))-h_{(\mathcal{X}, \overline{\mathcal{L}})}(Y)\right| \in \mathbb{R},
$$

where $Y$ runs the set of all subvarieties of $X_{\bar{K}}$. For $f=\left(f_{i}\right)_{i=1}^{\infty} \in \prod_{i=1}^{\infty} \mathcal{H}$, we set

$$
\widetilde{c}(\boldsymbol{f}):=\sup _{i \geq 1} \widetilde{c}\left(f_{i}\right) \in \mathbb{R} \cup\{+\infty\} .
$$

We define $\widetilde{\mathcal{B}}:=\left\{\boldsymbol{f}=\left(f_{i}\right)_{i=1}^{\infty} \in \prod_{i=1}^{\infty} \mathcal{H} \mid \widetilde{c}(\boldsymbol{f})<+\infty\right\}$. Note that, by virtue of Theorem 1.3, the property that $f$ belongs to $\widetilde{\mathcal{B}}$ is independent of the choice of $C^{\infty}$ models $(\mathcal{X}, \overline{\mathcal{L}})$ of $(X, L)$.

Theorem 4.2. Let $X$ a projective variety over a number field $K$, and $L$ an ample line bundle on $X$. Let $(\mathcal{X}, \overline{\mathcal{L}})$ be a $C^{\infty}$ model of $(X, L)$.

(1) There is a unique way to attach to each sequence $\boldsymbol{f}=\left(f_{i}\right)_{i=1}^{\infty} \in \widetilde{\mathcal{B}}$

$$
\widehat{h}_{L, f}:\left\{\text { subvariety of } X_{\bar{K}}\right\} \rightarrow \mathbb{R}
$$

with the following properties:

(i) For any subvariety $Y$ of $X_{\bar{K}}$, one has $\left|\widehat{h}_{L, \boldsymbol{f}}(Y)-h_{(\mathcal{X}, \overline{\mathcal{L}})}(Y)\right| \leq 2 \widetilde{c}(\boldsymbol{f})$;

(ii) $\widehat{h}_{L, S(\boldsymbol{f})} \circ f_{1}=d_{f_{1}} \widehat{h}_{L, \boldsymbol{f}}$.

(2) Moreover, $\widehat{h}_{L, f}$ is independent of the choice of $C^{\infty}$ models $(\mathcal{X}, \overline{\mathcal{L}})$ of $(X, L)$, and satisfies the following properties:

(iii) $\widehat{h}_{L, \boldsymbol{f}}(Y) \geq 0$ for any subvariety $Y$ of $X_{\bar{K}}$;

(iv) If $\left\{Y, f_{1}(Y), f_{2}\left(f_{1}(Y)\right), \cdots\right\}$ is a finite set, then $\widehat{h}_{L, \boldsymbol{f}}(Y)=0$;

(v) If $Y$ is a closed point, then $\widehat{h}_{L, \boldsymbol{f}}(Y)$ coincides with the one constructed in Theorem 2.3.

We call $\widehat{h}_{L, \boldsymbol{f}}(Y)$ a canonical height (normalized height) of $Y$ for $\boldsymbol{f}$.

Proof. Since we can prove Theorem 4.2(1) just as in Theorem 2.3, we only sketch a proof here. To construct $\widehat{h}_{L, f}(Y)$, we put

$$
h_{i}(Y):=\frac{1}{\prod_{\alpha=1}^{i} d_{f_{\alpha}}} h_{(\mathcal{X}, \overline{\mathcal{L}})}\left(f_{i} \circ f_{i-1} \circ \cdots \circ f_{1}(Y)\right) .
$$

Then $\left\{h_{i}(Y)\right\}_{i=0}^{\infty}$ is a Cauchy sequence, which allows one to define $\widehat{h}_{L, \boldsymbol{f}}(Y)=\lim _{i \rightarrow \infty} h_{i}(Y)$. One can check that $\widehat{h}_{L, f}$ satisfies (i) and (ii), and is unique.

We will show (2). By virtue of Theorem 1.3, it follows (4.1) that $\widehat{h}_{L, f}$ is independent of the choice of $C^{\infty}$ models of $(X, L)$. We take a $C^{\infty}$ model $(\mathcal{X}, \overline{\mathcal{L}})$ of $(X, L)$ such that $\mathcal{L}$ is ample, $c_{1}\left(\bar{L}_{\sigma}\right)$ is semipositive on $X_{\sigma}(\mathbb{C})$ for each $\sigma: K \rightarrow \mathbb{C}$, and $H^{0}\left(\mathcal{X}, \mathcal{L}^{\otimes n}\right)$ is generated by $\left\{s \in H^{0}\left(\mathcal{X}, \mathcal{L}^{\otimes n}\right) \mid\|s\|_{\text {sup }}<1\right\}$ (cf. [14, Lemma 1.3]). Then, for any $i \geq 1$, $h_{i}(Y) \geq 0$ for this model. Then we get (iii) by letting $i$ to the infinity. The finiteness of $\left\{Y, f_{1}(Y), f_{2}\left(f_{1}(Y)\right), \cdots\right\}$ implies that of $\left\{h_{(\mathcal{X}, \overline{\mathcal{L}})}(Y), h_{(\mathcal{X}, \overline{\mathcal{L}})}\left(f_{1}(Y)\right), h_{(\mathcal{X}, \overline{\mathcal{L}})}\left(f_{2}\left(f_{1}(Y)\right)\right), \cdots\right\}$. By (i) and (ii), we have

$$
\left|d_{f_{1}} \cdots d_{f_{i}} \widehat{h}_{L, \boldsymbol{f}}(Y)-h_{(\mathcal{X}, \overline{\mathcal{L}})}\left(f_{i} \circ \cdots \circ f_{1}(Y)\right)\right| \leq 2 \widetilde{c}\left(S^{i}(\boldsymbol{f})\right) \leq 2 \widetilde{c}(\boldsymbol{f})
$$

for any $i \geq 1$. Thus we get (iv). By (4.1), we get (v). 


\section{CANONical heights For Subvarieties, II}

In this section, we give another construction of a canonical height of subvarieties, using the adelic intersection theory in Zhang [20]. This construction will be used in $\S 7$.

Let $\mathcal{X}$ be a projective arithmetic variety and $\overline{\mathcal{L}}=\left(\mathcal{L},\left\{\|\cdot\|_{\sigma}\right\}_{\sigma}\right)$ a $C^{\infty}$ hermitian $\mathbb{Q}$-line bundle. Following $[14, \S 1]$, we say that $\overline{\mathcal{L}}$ is nef if $\mathrm{c}_{1}\left(\mathcal{L} \otimes_{K^{\sigma}} \mathbb{C}\right)$ is semipositive on $\mathcal{X}_{\sigma}(\mathbb{C})$ for all embeddings $\sigma: K \hookrightarrow \mathbb{C}$, and $\widehat{\operatorname{deg}}\left(\left.\overline{\mathcal{L}}\right|_{\Gamma}\right) \geq 0$ for all one-dimensional integral closed subschemes $\Gamma$ of $\mathcal{X}$. We say that $\overline{\mathcal{L}}$ is $\mathbb{Q}$-effective if there is a positive integer $n$ and a non-zero section $s \in H^{0}\left(\mathcal{X}, \mathcal{L}^{\otimes n}\right)$ such that $\|s\|_{\sigma \text {,sup }} \leq 1$ for all $\sigma: K \hookrightarrow \mathbb{C}$. We write $\overline{\mathcal{L}} \succsim 0$ if $\overline{\mathcal{L}}$ is $\mathbb{Q}$-effective. Moreover, if $U$ is a non-empty Zariski open set of $\mathcal{X}$ with $\operatorname{div}(s) \subseteq \mathcal{X} \backslash U$, then we write $\overline{\mathcal{L}}{ }_{U} 0$. For two $C^{\infty}$ hermitian $\mathbb{Q}$-line bundles $\overline{\mathcal{L}}$ and $\overline{\mathcal{M}}$ on $\mathcal{X}$, we write $\overline{\mathcal{L}} \succsim \overline{\mathcal{M}}$ (resp. $\left.\overline{\mathcal{L}} \succsim_{U} \overline{\mathcal{M}}\right)$ if $\overline{\mathcal{L}} \otimes \overline{\mathcal{M}}^{\otimes-1} \succsim 0\left(\right.$ resp. $\left.\overline{\mathcal{L}} \otimes \overline{\mathcal{M}}^{\otimes-1} \succsim_{U} 0\right)$

In the rest of this section, let $X$ be a projective variety over a number field $K$, and $L$ an ample line bundle on $X$.

Following [20, §1] and [14, §3.1], we define an adelic sequence of $C^{\infty}$ models. We also define a bounded sequence of $C^{\infty}$ models.

Definition 5.1. A sequence of $C^{\infty}$ models $\left\{\left(\mathcal{X}_{i}, \overline{\mathcal{L}}_{i}\right)\right\}_{i=0}^{\infty}$ of $(X, L)$ is called an adelic sequence (resp. a bounded sequence) if it satisfies the following properties:

(i) $\overline{\mathcal{L}}_{i}$ is nef for all $i$;

(ii) There is a non-empty Zariski open set $U \subseteq \operatorname{Spec}\left(O_{K}\right)$ such that $\left.\mathcal{X}_{i}\right|_{U}=\left.\mathcal{X}_{j}\right|_{U}$ (which we denote by $\left.\mathcal{X}_{U}\right)$ and $\left.\mathcal{L}_{i}\right|_{U}=\left.\mathcal{L}_{j}\right|_{U}$ in $\operatorname{Pic}\left(\mathcal{X}_{U}\right) \otimes \mathbb{Q}$ for all $i$ and $j$; The open set $U$ is called a common base;

(iii) For each $i, j$, there are a projective arithmetic variety $\mathcal{X}_{i, j} \stackrel{\pi_{i, j}}{\longrightarrow} \operatorname{Spec}\left(O_{K}\right)$, birational morphisms $\mu_{i, j}^{i}: \mathcal{X}_{i, j} \rightarrow \mathcal{X}_{i}$ and $\mu_{i, j}^{j}: \mathcal{X}_{i, j} \rightarrow \mathcal{X}_{j}$, and a nef $C^{\infty}$ hermitian $\mathbb{Q}$-line bundle $\overline{D_{i, j}}$ on $\operatorname{Spec}\left(O_{K}\right)$ such that

$$
\pi_{i, j}^{*}\left({\overline{D_{i, j}}}^{\otimes-1}\right) \precsim_{\pi_{i, j}^{-1}(U)}\left(\mu_{i, j}^{i}\right)^{*}\left(\overline{\mathcal{L}}_{i}\right) \otimes\left(\mu_{i, j}^{j}\right)^{*}\left(\overline{\mathcal{L}}_{j}^{\otimes-1}\right) \precsim_{\pi_{i, j}^{-1}(U)} \pi_{i, j}^{*}\left(\overline{D_{i, j}}\right)
$$

and that $\widehat{\operatorname{deg}}\left(\overline{D_{i, j}}\right) \rightarrow 0$ as $i, j \rightarrow+\infty$ (resp. $\widehat{\operatorname{deg}}\left(\overline{D_{i, j}}\right)$ is bounded with respect to $i, j)$.

Let $\left\{\left(\mathcal{X}_{i}, \overline{\mathcal{L}}_{i}\right)\right\}_{n=0}^{\infty}$ be an adelic sequence (resp. bounded sequence) of $C^{\infty}$ models of $(X, L)$. Let $g: Y \rightarrow X$ is a finite morphism of projective varieties over $K$. Suppose, for each $n$, we are given a morphism $g_{i}: \mathcal{Y}_{i} \rightarrow \mathcal{X}_{i}$ of projective arithmetic varieties over $\operatorname{Spec}\left(O_{K}\right)$ that extends $g: Y \rightarrow X$ such that $\left.\mathcal{Y}_{i}\right|_{U}=\left.\mathcal{Y}_{j}\right|_{U}$ and $\left.g_{i}\right|_{U}=\left.g_{j}\right|_{U}$ for all $i$ and $j$. As mentioned in $\left[14\right.$, p. 41] we can then see that $\left\{\left(\mathcal{Y}_{i}, g_{i}^{*}\left(\overline{\mathcal{L}}_{i}\right)\right)\right\}_{n=0}^{\infty}$ is an adelic sequence (resp. bounded sequence) of $C^{\infty}$ models of $\left(Y, g^{*}(L)\right)$.

One has the following theorem ([20, Theorem (1.4)] and [14, Proposition 4.1.1]).

Theorem $5.2([20],[14])$. Let $\left\{\left(\mathcal{X}_{i}, \overline{\mathcal{L}}_{i}^{(n)}\right)\right\}_{i=0}^{\infty}$ be adelic sequences of $C^{\infty}$ models of $(X, L)$ for $n=1,2, \ldots, \operatorname{dim} X+1$. Then the arithmetic intersection number

$$
\widehat{\operatorname{deg}}\left(\widehat{\mathrm{c}}_{1}\left(\overline{\mathcal{L}}_{i}^{(1)}\right) \widehat{\mathrm{c}}_{1}\left(\overline{\mathcal{L}}_{i}^{(2)}\right) \cdots \widehat{\mathrm{c}}_{1}\left(\overline{\mathcal{L}}_{i}^{(\operatorname{dim} X+1)}\right)\right)
$$

converges as $i$ tends to $+\infty$. 
For $i=1,2, \ldots$, let $f_{i}: X \rightarrow X$ be a morphism over $K$ with $f_{i}^{*}(L) \simeq L^{\otimes d_{f_{i}} \text { for some }}$ integer $d_{f_{i}} \geq 2$. Since we assume $L$ is ample, every $f_{i}$ is finite. We say that a sequence $\boldsymbol{f}=\left(f_{i}\right)_{i=1}^{\infty}$ is adelically bounded if there are a $C^{\infty} \operatorname{model}(\mathcal{X}, \overline{\mathcal{L}})$ of $(X, L)$ such that $\overline{\mathcal{L}}$ is nef, and a non-empty Zariski open set $U \subseteq \operatorname{Spec}\left(O_{K}\right)$ with the following properties:

(i) $f_{i}: X \rightarrow X$ extend to $f_{i U}: \mathcal{X}_{U} \rightarrow \mathcal{X}_{U}$ for each $i$, where $\mathcal{X}_{U}=\left.\mathcal{X}\right|_{U}$;

(ii) Let $\mathcal{Z}_{i}$ be the normalization of $\mathcal{X}_{U} \stackrel{f_{i U}}{\longrightarrow} \mathcal{X}_{U} \hookrightarrow \mathcal{X}$; We write $\widetilde{f}_{i}$ for the induced morphism $\widetilde{f}_{i}: \mathcal{Z}_{i} \rightarrow \mathcal{X}$, and set $\overline{\mathcal{N}}_{i}=\widetilde{f}_{i}^{*}(\overline{\mathcal{L}})^{\otimes \frac{1}{d_{f_{i}}}} ;$ We also put $\mathcal{Z}_{0}=\mathcal{X}$ and $\overline{\mathcal{N}}_{0}=\overline{\mathcal{L}}$; Then $\left\{\left(\mathcal{Z}_{i}, \overline{\mathcal{N}}_{i}\right)\right\}_{i=0}^{\infty}$ is a bounded sequence with the common base $U$.

For example, it is easy to see that an iteration by a finite number of morphisms $g_{1}, \ldots, g_{k}$ in Example 2.1 gives an adelically bounded sequence.

Let us make an adelic sequence of $C^{\infty}$ models from an adelically bounded sequence.

Proposition 5.3. Let $\boldsymbol{f}=\left(f_{i}\right)_{i=1}^{\infty}$ be adelically bounded, and $(\mathcal{X}, \overline{\mathcal{L}})$ and $U$ in the definition of an adelically bounded sequence. Let $\mathcal{X}_{i}$ be the normalization of

$$
\mathcal{X}_{U} \stackrel{f_{1 U}}{\longrightarrow} \mathcal{X}_{U} \stackrel{f_{2 U}}{\longrightarrow} \cdots \stackrel{f_{i U}}{\longrightarrow} \mathcal{X}_{U} \hookrightarrow \mathcal{X}
$$

We write $F_{i}$ for the induced morphism $F_{i}: \mathcal{X}_{i} \rightarrow \mathcal{X}$, and set $\overline{\mathcal{L}}_{i}=F_{i}^{*}(\overline{\mathcal{L}})^{\otimes \frac{1}{d_{f_{1}} \cdots d_{f_{i}}}}$. We also put $\mathcal{X}_{0}=\mathcal{X}$ and $\overline{\mathcal{L}}_{0}=\overline{\mathcal{L}}$. Then $\left\{\left(\mathcal{X}_{i}, \overline{\mathcal{L}}_{i}\right)\right\}_{i=0}^{\infty}$ is an adelic sequence.

Proof. Let $\left\{\left(\mathcal{Z}_{i}, \overline{\mathcal{N}}_{i}\right)\right\}_{i=0}^{\infty}$ be the bounded sequence of $C^{\infty}$ models and $\tilde{f}_{i}: \mathcal{Z}_{i} \rightarrow \mathcal{X}$ the morphisms in the definition of an adelically bounded sequence.

Step. 1: We compare $\left(\mathcal{X}_{i}, \overline{\mathcal{L}}_{i}\right)$ with $\left(\mathcal{X}_{i+1}, \overline{\mathcal{L}}_{i+1}\right)$. Since $\mathcal{Z}_{i+1}$ is the normalization of $\mathcal{X}_{U} \stackrel{f_{i+1 U}}{\longrightarrow} \mathcal{X}_{U} \hookrightarrow \mathcal{X}$, we see that $\mathcal{X}_{i+1}$ is the normalization of

$$
\mathcal{X}_{U} \stackrel{f_{1 U}}{\longrightarrow} \mathcal{X}_{U} \stackrel{f_{2 U}}{\longrightarrow} \cdots \stackrel{f_{i U}}{\longrightarrow} \mathcal{X}_{U} \hookrightarrow \mathcal{Z}_{i+1}
$$

We write $G_{i}: \mathcal{X}_{i+1} \rightarrow \mathcal{Z}_{i+1}$ for the induced morphism. Since $F_{i+1}$ and $\tilde{f}_{i+1} \circ G_{i}$ are both morphisms form $\mathcal{X}_{i+1}$ to $\mathcal{X}$ and coincides over $U$, we get $F_{i+1}=\widetilde{f}_{i+1} \circ G_{i}$. Then

$$
\overline{\mathcal{L}}_{i+1}=F_{i+1}^{*}(\overline{\mathcal{L}})^{\otimes \frac{1}{d_{f_{1} \cdots d_{f_{i}} d_{f_{i+1}}}}}=\left(G_{i}\right)^{*}\left(\widetilde{f}_{i+1}\right)^{*}(\overline{\mathcal{L}})^{\otimes \frac{1}{d_{f_{1} \cdots d_{f_{i}} d_{f_{i+1}}}}}=\left(G_{i}\right)^{*}\left(\overline{\mathcal{N}_{i+1}}\right)^{\otimes \frac{1}{d_{f_{1}} \cdots d_{f_{i}}}}
$$

Since $\left\{\left(\mathcal{Z}_{i}, \overline{\mathcal{N}}_{i}\right)\right\}_{i=0}^{\infty}$ is a bounded sequence, there are a projective arithmetic variety $\mathcal{W}_{i+1} \stackrel{\pi_{i+1}}{\longrightarrow}$ $\operatorname{Spec}\left(O_{K}\right)$, birational morphisms $\mu_{i+1}: \mathcal{W}_{i+1} \rightarrow \mathcal{Z}_{0}=\mathcal{X}$ and $\nu_{i+1}: \mathcal{W}_{i+1} \rightarrow \mathcal{Z}_{i+1}$, and a nef $C^{\infty}$ hermitian $\mathbb{Q}$-line bundle $\overline{D_{i+1}}$ on $\operatorname{Spec}\left(O_{K}\right)$ such that

$$
\pi_{i+1}^{*}\left({\overline{D_{i+1}}}^{\otimes-1}\right) \precsim_{\pi_{i+1}^{-1}(U)} \mu_{i+1}^{*}\left(\overline{\mathcal{N}}_{0}\right) \otimes \nu_{i+1}^{*}\left(\overline{\mathcal{N}}_{i+1}^{\otimes-1}\right) \precsim_{\pi_{i+1}^{-1}(U)} \pi_{i+1}^{*}\left(\overline{D_{i+1}}\right)
$$

and that there is a constant $C$ such that $0 \leq \widehat{\operatorname{deg}}\left(\overline{D_{i+1}}\right) \leq C$ for all $i$.

There is a projective arithmetic variety $\mathcal{W}_{i+1}^{\prime} \stackrel{\pi_{i+1}^{\prime}}{\longrightarrow} \operatorname{Spec}\left(O_{K}\right)$, birational morphisms $\mu_{i+1}^{\prime}$ : $\mathcal{W}_{i+1}^{\prime} \rightarrow \mathcal{X}_{i}$ and $\nu_{i+1}^{\prime}: \mathcal{W}_{i+1}^{\prime} \rightarrow \mathcal{X}_{i+1}$ that are the identity map over $U$, and a morphism $H_{i}: \mathcal{W}_{i+1}^{\prime} \rightarrow \mathcal{W}_{i+1}$ that extends $f_{1 U} \circ \cdots \circ f_{i U}$. Indeed, let $\mathcal{W}_{i+1}^{\prime \prime}$ be the normalization of $\mathcal{X}_{U} \stackrel{f_{1 U}}{\longrightarrow} \mathcal{X}_{U} \stackrel{f_{2 U}}{\longrightarrow} \cdots \stackrel{f_{i U}}{\longrightarrow} \mathcal{X}_{U} \hookrightarrow \mathcal{W}_{i+1}$, and we may take $\mathcal{W}_{i+1}^{\prime}$ as the Zariski closure of $\mathcal{X}_{U}$ 
diagonally embedded in $\mathcal{X}_{i} \times_{\operatorname{Spec}\left(O_{K}\right)} \mathcal{W}_{i+1}^{\prime \prime} \times_{\operatorname{Spec}\left(O_{K}\right)} \mathcal{X}_{i+1}$. Thus we get the following diagram:

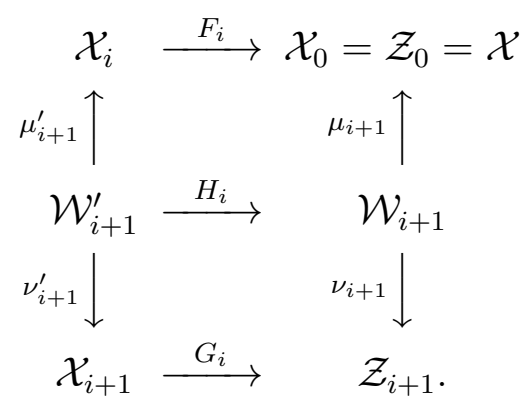

Since $F_{i} \circ \mu_{i+1}^{\prime}$ and $\mu_{i+1} \circ H_{i}$ are both morphisms from $\mathcal{W}_{i+1}^{\prime}$ to $\mathcal{X}_{0}$ and coincide over $U$, we get $F_{i} \circ \mu_{i+1}^{\prime}=\mu_{i+1} \circ H_{i}$. Similarly $G_{i} \circ \nu_{i+1}^{\prime}=\nu_{i+1} \circ H_{i}$. By (5.1), we have

$$
\begin{aligned}
\left(\mu_{i+1}^{\prime}\right)^{*}\left(\overline{\mathcal{L}}_{i}\right) \otimes\left(\nu_{i+1}^{\prime}\right)^{*}\left(\overline{\mathcal{L}}_{i+1}^{\otimes-1}\right) & =\left(\mu_{i+1}^{\prime}\right)^{*}\left(F_{i}^{*}\left(\overline{\mathcal{L}_{0}}\right)^{\otimes \frac{1}{d_{f_{1}} \cdots d_{f_{i}}}}\right) \otimes\left(\nu_{i+1}^{\prime}\right)^{*}\left(G_{i}^{*}\left(\overline{\mathcal{N}_{i+1}}\right)^{\otimes-\frac{1}{d_{f_{1}} \cdots d_{f_{i}}}}\right) \\
& =H_{i}^{*}\left(\mu_{i+1}^{*}\left(\overline{\mathcal{N}}_{0}\right)^{\otimes \frac{1}{d_{f_{1} \cdots d_{f_{i}}}}} \otimes \nu_{i+1}^{*}\left(\overline{\mathcal{N}}_{i+1}\right)^{\otimes-\frac{1}{d_{f_{1} \cdots d_{f_{i}}}}}\right) .
\end{aligned}
$$

Then by (5.2) we get

$$
\left(\pi_{i+1}^{\prime}\right)^{*}\left(\overline{D_{i+1}}\right)^{\otimes-\frac{1}{d_{f_{1} \cdots d_{f_{i}}}}} \precsim_{\pi_{i+1}^{\prime-1}(U)}\left(\mu_{i+1}^{\prime}\right)^{*}\left(\overline{\mathcal{L}}_{i}\right) \otimes\left(\nu_{i+1}^{\prime}\right)^{*}\left(\overline{\mathcal{L}}_{i+1}^{\otimes-1}\right) \precsim_{\pi_{i+1}^{\prime-1}(U)}\left(\pi_{i+1}^{\prime}\right)^{*}\left(\overline{D_{i+1}}\right)^{\otimes \frac{1}{d_{f_{1} \cdots d_{f_{i}}}}} .
$$

Step. 2: For $i>j \geq 0$, we compare $\left(\mathcal{X}_{i}, \overline{\mathcal{L}}_{i}\right)$ with $\left(\mathcal{X}_{j}, \overline{\mathcal{L}}_{j}\right)$. We take a projective arithmetic variety $\mathcal{W} \stackrel{\pi}{\longrightarrow} \operatorname{Spec}\left(O_{K}\right)$ such that, for each $\alpha=i+1, \ldots, j$, there is a birational morphism $p_{\alpha}: \mathcal{W} \rightarrow \mathcal{W}_{\alpha}^{\prime}$ that is the identity map over $U$. Indeed, we may take $\mathcal{W}$ as the Zariski closure of $\mathcal{X}_{U}$ diagonally embedded in $\mathcal{W}_{i+1}^{\prime} \times_{\operatorname{Spec}\left(O_{K}\right)} \mathcal{W}_{i+2}^{\prime} \times_{\operatorname{Spec}\left(O_{K}\right)} \cdots \times_{\operatorname{Spec}\left(O_{K}\right)} \mathcal{W}_{j}^{\prime}$. For $\alpha=i+1, \ldots, j$, we define the birational morphism $\xi_{\alpha}: \mathcal{W} \rightarrow \mathcal{X}_{\alpha}$ by

$$
\begin{aligned}
& \xi_{i}=\mu_{i+1}^{\prime} \circ p_{i+1}, \quad \xi_{i+1}=\mu_{i+2}^{\prime} \circ p_{i+2}=\nu_{i+1}^{\prime} \circ p_{i+1}, \ldots, \\
& \xi_{j-1}=\mu_{j}^{\prime} \circ p_{j}=\nu_{j-1}^{\prime} \circ p_{j-1}, \quad \xi_{j}=\nu_{j}^{\prime} \circ p_{j} .
\end{aligned}
$$

Here $\mu_{\alpha+1}^{\prime} \circ p_{\alpha+1}=\nu_{\alpha}^{\prime} \circ p_{\alpha}$ for $\alpha=i+1, \ldots, j-1$ because $\mu_{\alpha+1}^{\prime} \circ p_{\alpha+1}$ and $\nu_{\alpha}^{\prime} \circ p_{\alpha}$ are the identity map over $U$ whence over $\operatorname{Spec}\left(O_{K}\right)$.

In what follows, we write the group structure of the Picard group additively. By (5.3), we have

$$
-\frac{1}{d_{f_{1}} \cdots d_{f_{\alpha}}} \pi^{*}\left(\overline{D_{\alpha+1}}\right) \precsim_{\pi^{-1}(U)} \xi_{\alpha}^{*}\left(\overline{\mathcal{L}}_{\alpha}\right)-\xi_{\alpha+1}^{*}\left(\overline{\mathcal{L}}_{\alpha+1}\right) \precsim_{\pi^{-1}(U)} \frac{1}{d_{f_{1}} \cdots d_{f_{\alpha}}} \pi^{*}\left(\overline{D_{\alpha+1}}\right) .
$$

Since $\xi_{i}^{*}\left(\overline{\mathcal{L}}_{i}\right)-\xi_{j}^{*}\left(\overline{\mathcal{L}}_{j}\right)=\sum_{\alpha=i}^{j-1}\left(\xi_{\alpha}^{*}\left(\overline{\mathcal{L}}_{\alpha}\right)-\xi_{\alpha+1}^{*}\left(\overline{\mathcal{L}}_{\alpha+1}\right)\right)$, we get

$$
-\sum_{\alpha=i}^{j-1} \frac{1}{d_{f_{1}} \cdots d_{f_{\alpha}}} \pi^{*}\left(\overline{D_{\alpha+1}}\right) \precsim_{\pi^{-1}(U)} \xi_{i}^{*}\left(\overline{\mathcal{L}}_{i}\right)-\xi_{j}^{*}\left(\overline{\mathcal{L}}_{j}\right) \precsim_{\pi^{-1}(U)}^{j-1} \sum_{\alpha=i} \frac{1}{d_{f_{1}} \cdots d_{f_{\alpha}}} \pi^{*}\left(\overline{D_{\alpha+1}}\right) .
$$

Since $0 \leq \widehat{\operatorname{deg}}\left(\overline{D_{\alpha+1}}\right) \leq C$ for all $\alpha$, we have $\sum_{\alpha=i}^{j-1} \frac{1}{d_{f_{1} \cdots d_{f_{\alpha}}}} \widehat{\operatorname{deg}}\left(\overline{D_{\alpha+1}}\right) \rightarrow 0$ as $i, j$ tends to $+\infty$. Thus $\left\{\left(\mathcal{X}_{i}, \overline{\mathcal{L}}_{i}\right)\right\}_{i=0}^{\infty}$ is an adelic sequence. 
To give another construction of canonical heights for subvarieties, we need the following proposition ([14, Proposition 2.1 and its proof $]$ ).

Proposition $5.4([14])$. Let $\left(\mathcal{X}_{1}, \overline{\mathcal{L}}_{1}\right)$ and $\left(\mathcal{X}_{2}, \overline{\mathcal{L}}_{2}\right)$ be two $C^{\infty}$ models of $(X, L)$ such that $\overline{\mathcal{L}}_{1}$ and $\overline{\mathcal{L}}_{2}$ are nef. Assume that there are a non-empty Zariski open set $U \subseteq \operatorname{Spec}\left(O_{K}\right)$, a projective arithmetic variety $\mathcal{X}_{3} \stackrel{\pi}{\longrightarrow} \operatorname{Spec}\left(O_{K}\right)$, birational morphisms $\mu_{1}: \mathcal{X}_{3} \rightarrow \mathcal{X}_{1}$ and $\mu_{2}: \mathcal{X}_{3} \rightarrow \mathcal{X}_{2}$, and a nef $C^{\infty}$ hermitian $\mathbb{Q}$-line bundle $\bar{D}$ on $\operatorname{Spec}\left(O_{K}\right)$, such that

$$
\pi^{*}\left(\bar{D}^{\otimes-1}\right) \precsim_{\pi^{-1}(U)} \mu_{1}^{*}\left(\overline{\mathcal{L}}_{1}\right) \otimes \mu_{2}^{*}\left(\overline{\mathcal{L}}_{2}^{\otimes-1}\right) \precsim_{\pi^{-1}(U)} \pi^{*}(\bar{D}) .
$$

Let $Y$ be a subvariety of $X_{\bar{K}}$, which is defined over a finite extension field $K^{\prime}$ of $K$. Let $\mathcal{Y}$ be the Zariski closure of $\mathcal{X}_{3} \times_{\operatorname{Spec}\left(O_{K}\right)} \operatorname{Spec}\left(O_{K}^{\prime}\right)$. Let $p: \mathcal{X}_{3} \times_{\operatorname{Spec}\left(O_{K}\right)} \operatorname{Spec}\left(O_{K}^{\prime}\right) \rightarrow \mathcal{X}_{3}$ be the natural morphism. Then

$$
\begin{aligned}
\mid \widehat{\operatorname{deg}}\left(\widehat{\mathrm{c}}_{1}\left(\left.\left(\mu_{1} \circ p\right)^{*}\left(\overline{\mathcal{L}}_{1}\right)\right|_{\mathcal{Y}}\right)^{\cdot \operatorname{dim} Y+1}\right)-\widehat{\operatorname{deg}}( & \left.\widehat{\mathrm{c}}_{1}\left(\left.\left(\mu_{2} \circ p\right)^{*}\left(\overline{\mathcal{L}}_{2}\right)\right|_{\mathcal{Y}}\right)^{\cdot \operatorname{dim} Y+1}\right) \mid \\
& \leq\left[K^{\prime}: K\right](\operatorname{dim} Y+1) \operatorname{deg}\left(\left.L\right|_{Y} ^{\operatorname{dim} Y}\right) \widehat{\operatorname{deg}}(\bar{D}) .
\end{aligned}
$$

Proposition 5.5. Let $\boldsymbol{f}=\left(f_{i}\right)_{i=1}^{\infty}$ be adelically bounded, and $\left\{\left(\mathcal{X}_{i}, \overline{\mathcal{L}}_{i}\right)\right\}_{i=0}^{\infty}$ the adelic sequence of $C^{\infty}$ models in Proposition 5.3. Then for any subvariety $Y$ of $X_{\bar{K}}$, we have

$$
\widehat{h}_{L, f}(Y)=\lim _{i \rightarrow \infty} h_{\left(\mathcal{X}_{i}, \overline{\mathcal{L}}_{i}\right)}(Y) \text {. }
$$

Moreover, the convergence is uniform with respect to $Y$.

Proof. We first check that $\boldsymbol{f}$ belongs to $\widetilde{\mathcal{B}}$ so that $\widehat{h}_{L, f}(Y)$ is well-defined. Let $\left\{\left(\mathcal{Z}_{i}, \overline{\mathcal{N}}_{i}\right)\right\}_{i=0}^{\infty}$ be the bounded sequence of $C^{\infty}$ models in the definition of an adelically bounded sequence. By the projection formula, we have $h_{\left(\mathcal{Z}_{i}, \overline{\mathcal{N}}_{i}\right)}(Y)=\frac{1}{d_{f_{i}}} h_{\left(\mathcal{Z}_{0}, \overline{\mathcal{N}}_{0}\right)}\left(f_{i}(Y)\right)$. On the other hand, it follows from the projection formula, Proposition 5.4 and the definition of an bounded sequence of $C^{\infty}$ models that there is a constant $C$ independent of $i$ and $Y$ such that

$$
\left|h_{\left(\mathcal{Z}_{i}, \bar{N}_{i}\right)}(Y)-h_{\left(\mathcal{Z}_{0}, \bar{N}_{0}\right)}(Y)\right| \leq C .
$$

Since $\left(\mathcal{Z}_{0}, \overline{\mathcal{N}}_{0}\right)=(\mathcal{X}, \overline{\mathcal{L}})$, we get $\left|\frac{1}{d_{f_{i}}} h_{(\mathcal{X}, \overline{\mathcal{L}})}\left(f_{i}(Y)\right)-h_{(\mathcal{X}, \overline{\mathcal{L}})}(Y)\right| \leq C$ for all $i$ and $Y$. Thus $\boldsymbol{f}$ belongs to $\widetilde{\mathcal{B}}$. For the right-hand side of $(5.4)$, it follows from the projection formula that

$$
h_{\left(\mathcal{X}_{i}, \overline{\mathcal{L}}_{i}\right)}(Y)=\frac{1}{d_{f_{1}} \cdots d_{f_{i}}} h_{(\mathcal{X}, \overline{\mathcal{L}})}\left(f_{i} \circ \cdots \circ f_{1}(Y)\right) .
$$

Then by (4.1), we get the first assertion. Since

$$
\begin{aligned}
& \left|h_{\left(\mathcal{X}_{i}, \overline{\mathcal{L}}_{i}\right)}(Y)-h_{\left(\mathcal{X}_{i+1}, \overline{\mathcal{L}}_{i+1}\right)}(Y)\right| \\
& =\left|\frac{1}{d_{f_{1}} \cdots d_{f_{i}}} h_{(\mathcal{X}, \overline{\mathcal{L}})}\left(f_{i} \circ \cdots \circ f_{1}(Y)\right)-\frac{1}{d_{f_{1}} \cdots d_{f_{i+1}}} h_{(\mathcal{X}, \overline{\mathcal{L}})}\left(f_{i+1} \circ \cdots \circ f_{1}(Y)\right)\right| \leq \frac{C}{d_{f_{1}} \cdots d_{f_{i}}},
\end{aligned}
$$

the convergence is uniform with respect to $Y$. 


\section{Admissible Metrics}

In this section, we consider a local theory in the setting of $[20, \S 2]$, and in case $X=\mathbb{P}^{N}$ over $\mathbb{C}$, we see its relation with Green currents in [9].

Let $K_{v}$ be an algebraically closed valuation field, $X$ a projective variety over $K_{v}$, and $L$ a line bundle on $X$. Let $\|\cdot\|$ be a continuous and bounded metric on $L$. (When $K_{v}$ is nonarchimedean, we refer to $[20,(1.1)]$ for the definition of $\|\cdot\|$ being a continuous and bounded metric.)

We set

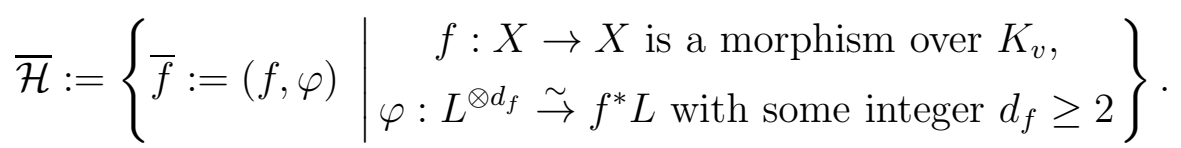

For $\bar{f}=(f, \varphi) \in \overline{\mathcal{H}}$, we set

$$
\bar{c}(\bar{f})=\sup _{x \in X\left(K_{v}\right)}\left|\log \frac{\left(\varphi^{*} f^{*}\|\cdot\|\right)^{\frac{1}{d_{f}}}}{\|\cdot\|}(x)\right| \in \mathbb{R} .
$$

Moreover, for $\overline{\boldsymbol{f}}=\left(\overline{f_{i}}\right)_{i=1}^{\infty} \in \prod_{i=1}^{\infty} \overline{\mathcal{H}}$, we set

$$
\bar{c}(\overline{\boldsymbol{f}})=\sup _{i \geq 1} \bar{c}\left(\overline{f_{i}}\right) \quad \in \mathbb{R} \cup\{+\infty\}
$$

We define $\overline{\mathcal{B}}=\left\{\bar{f} \in \prod_{i=1}^{\infty} \overline{\mathcal{H}} \mid \bar{c}(\bar{f})<+\infty\right\}$. Note that the property that $\bar{f}$ belongs to $\overline{\mathcal{B}}$ is independent of the choice of bounded and continuous metrics $\|\cdot\|$.

Let $S: \prod_{i=1}^{\infty} \overline{\mathcal{H}} \rightarrow \prod_{i=1}^{\infty} \overline{\mathcal{H}}$ be the shift map defined by $S\left(\left(\overline{f_{i}}\right)_{i=1}^{\infty}\right)=\left(\overline{f_{i+1}}\right)_{i=1}^{\infty}$. It follows from $\bar{c}(S(\bar{f})) \leq \bar{c}(\bar{f})$ that $S$ sends $\overline{\mathcal{B}}$ to $\overline{\mathcal{B}}$.

Theorem 6.1. Let $X$ be a projective variety over an algebraically closed valuation field $K_{v}$, and $L$ a line bundle on $X$. Let $\|\cdot\|$ be a continuous and bounded metric on $L$. Then there is a unique way to attach to each $\bar{f} \in \overline{\mathcal{B}}$ a metric $\widehat{\|\cdot\|}_{L, \bar{f}}$ with the following properties:

(i) The metric $\widehat{\|\cdot\|}_{L, \bar{f}}$ is bounded and continuous such that

$$
\sup _{x \in X\left(K_{v}\right)}\left|\log \frac{\widehat{\|\cdot\|}_{L, \bar{f}}}{\|\cdot\|}(x)\right| \leq 2 \bar{c}(\bar{f})
$$

(ii) $\varphi_{1}^{*} f_{1}^{*} \widehat{\|\cdot\|_{L, S(\overline{\boldsymbol{f}})}}=\widehat{\|\cdot\|_{L, \bar{f}}} d_{f_{1}}$

Moreover, the metric $\widehat{\|\cdot\|}_{L, \bar{f}}$ is independent of the choice of bounded and continuous metrics on $L$. We call $\widehat{\|\cdot\|}_{L, \overline{\boldsymbol{f}}}$ the admissible metric for $\overline{\boldsymbol{f}}$.

Proof. We denote by $g_{i}$ the bounded and continuous function

$$
g_{i}:=\log \frac{\left(\varphi_{i}^{*} f_{i}^{*}\|\cdot\|\right)^{\frac{1}{d_{f_{i}}}}}{\|\cdot\|}
$$


on $X\left(K_{v}\right)$. We define the bounded and continuous metric $\|\cdot\|_{i}$ on $L$ by

$$
\begin{aligned}
\|\cdot\|_{0} & :=\|\cdot\|, \\
\|\cdot\|_{i} & :=\left(\varphi_{1}^{*} f_{1}^{*} \cdots\left(\varphi_{i-1}^{*} f_{i-1}^{*}\left(\varphi_{i}^{*} f_{i}^{*}\|\cdot\|\right)^{\frac{1}{d_{f_{i}}}}\right)^{\frac{1}{f_{f_{i-1}}}} \ldots\right)^{\frac{1}{d_{f_{1}}}} \quad(\text { for } i \geq 1) .
\end{aligned}
$$

Then

$$
\log \frac{\|\cdot\|_{i}}{\|\cdot\|_{i-1}}=\prod_{\beta=1}^{i-1}\left(\frac{1}{d_{f_{\beta}}} \varphi_{\beta}^{*} f_{\beta}^{*}\right)\left[\frac{1}{d_{f_{i}}} \varphi_{i}^{*} f_{i}^{*}\left(\log \|\cdot\|_{0}\right)-\log \|\cdot\|_{0}\right]=\prod_{\beta=1}^{i-1}\left(\frac{1}{d_{f_{\beta}}} \varphi_{\beta}^{*} f_{\beta}^{*}\right) g_{i} .
$$

We then have

$$
\log \frac{\|\cdot\|_{i}}{\|\cdot\|_{0}}=\sum_{\alpha=1}^{i} \log \frac{\|\cdot\|_{\alpha}}{\|\cdot\|_{\alpha-1}}=\sum_{\alpha=1}^{i} \prod_{\beta=1}^{\alpha-1}\left(\frac{1}{d_{f_{\beta}}} \varphi_{\beta}^{*} f_{\beta}^{*}\right) g_{\alpha}
$$

Since $\sup _{x \in X\left(K_{v}\right)}\left|g_{\alpha}(x)\right| \leq \bar{c}(\bar{f})$ for any $\alpha \geq 1$, it follows that

$$
\sup _{x \in X\left(K_{v}\right)}\left|\sum_{\alpha=1}^{i} \prod_{\beta=1}^{\alpha-1}\left(\frac{1}{d_{f_{\beta}}} \varphi_{\beta}^{*} f_{\beta}^{*}\right) g_{\alpha}(x)\right| \leq \sum_{\alpha=1}^{i} \frac{\bar{c}(\overline{\boldsymbol{f}})}{\prod_{\beta=1}^{\alpha-1} d_{f_{\beta}}} \leq \sum_{\alpha=1}^{i} \frac{\bar{c}(\overline{\boldsymbol{f}})}{2^{\alpha-1}} .
$$

Thus, as $i$ tends to the infinity, $\sum_{\alpha=1}^{i} \prod_{\beta=1}^{\alpha-1}\left(\frac{1}{d_{f_{\beta}}} \varphi_{\beta}^{*} f_{\beta}^{*}\right) g_{\alpha}$ converges to a bounded and continuous function, which we denote by $g_{\overline{\boldsymbol{f}}}$. We set $\|\cdot\|_{L, \overline{\boldsymbol{f}}}:=\|\cdot\|_{0} \exp \left(g_{\overline{\boldsymbol{f}}}\right)$.

Let us check $\widehat{\|\cdot\|}_{L, \bar{f}}$ satisfies (i) and (ii). Using (6.1), one finds

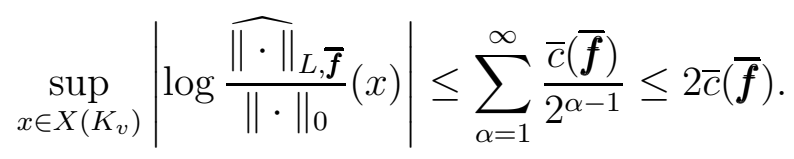

This shows (i). To see (ii), we first note

$$
\log \frac{\widehat{\|\cdot\|_{L, S(\overline{\boldsymbol{f}})}}}{\|\cdot\|_{0}}=\sum_{\alpha=1}^{\infty} \prod_{\beta=1}^{\alpha-1}\left(\frac{1}{d_{f_{\beta+1}}} \varphi_{\beta+1}^{*} f_{\beta+1}^{*}\right) g_{\alpha+1} .
$$

Then we get

$$
\begin{aligned}
\varphi_{1}^{*} f_{1}^{*}\left(\log \widehat{\|\cdot\|_{L, S(\overline{\boldsymbol{f}})}}\right) & =\varphi_{1}^{*} f_{1}^{*}\left(\log \|\cdot\|_{0}\right)+\varphi_{1}^{*} f_{1}^{*}\left(\sum_{\alpha=1}^{\infty} \prod_{\beta=1}^{\alpha-1}\left(\frac{1}{d_{f_{\beta+1}}} \varphi_{\beta+1}^{*} f_{\beta+1}^{*}\right) g_{\alpha+1}\right) \\
& =\varphi_{1}^{*} f_{1}^{*}\left(\log \|\cdot\|_{0}\right)-d_{f_{1}} g_{1}+d_{f_{1}}\left(\sum_{\alpha=1}^{\infty} \prod_{\beta=1}^{\alpha-1}\left(\frac{1}{d_{f_{\beta}}} \varphi_{\beta}^{*} f_{\beta}^{*}\right) g_{\alpha}\right) \\
& =\varphi_{1}^{*} f_{1}^{*}\left(\log \|\cdot\|_{0}\right)-d_{f_{1}} g_{1}+d_{f_{1}}\left(\log \widehat{\|\cdot\|_{L, \bar{f}}}-\log \|\cdot\|_{0}\right)=d_{f_{1}} \log \widehat{\|\cdot\|_{L, \overline{\boldsymbol{f}}}}
\end{aligned}
$$

where we used $g_{1}=\log \frac{\left(\varphi_{1}^{*} f_{1}^{*}\|\cdot\|\right)^{\frac{1}{d_{f_{1}}}}}{\|\cdot\|}$ in the last equality. This shows (ii). 
Next we show the uniqueness. Suppose $\left\{{\widehat{\|\cdot\|_{L, \bar{f}}^{\prime}}}^{\prime}\right.$ are metrics on $L$ satisfying (i) and (ii). Then we have by (ii)

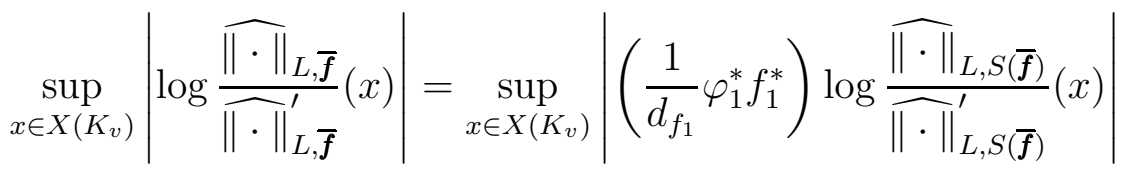

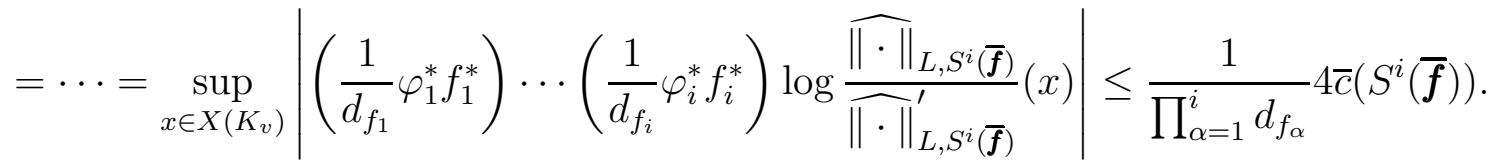

Since $\bar{c}\left(S^{i}(\overline{\boldsymbol{f}})\right) \leq \bar{c}(\overline{\boldsymbol{f}})$, letting $i$ to the infinity, we find $\widehat{\|\cdot\|}_{L, \overline{\boldsymbol{f}}}={\widehat{\|\cdot\|_{L, \overline{\boldsymbol{f}}}^{\prime}}}^{\prime}$

In the rest of this section, we show that $\widehat{\|\cdot\|}_{L, \bar{f}}$ is independent of the choice of $\|\cdot\|$. Since the property of $\bar{f} \in \overline{\mathcal{B}}$ is independent of the choice of $\|\cdot\|$, let us start from another bounded and continuous metric $\|\cdot\|^{\prime \prime}$ to obtain ${\widehat{\|\cdot\|_{L, \bar{f}}^{\prime \prime}}}^{\prime \prime}$ Set $\bar{c}=\sup _{x \in X\left(K_{v}\right)}\left|\log \frac{\|\cdot\|^{\prime \prime}}{\|\cdot\|}\right|$. Then

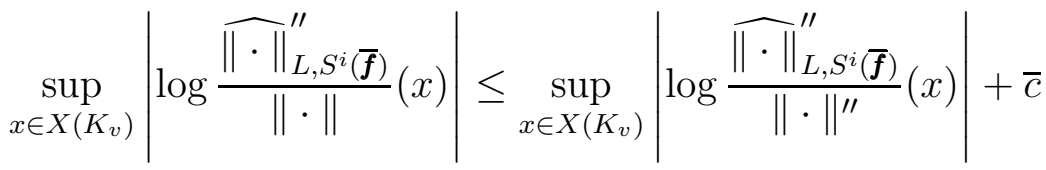

$$
\begin{aligned}
& \leq 2 \bar{c}\left(S^{i}(\overline{\boldsymbol{f}})\right)+5 \bar{c} \leq 2 \bar{c}(\overline{\boldsymbol{f}})+5 \bar{c}
\end{aligned}
$$

for any $i \geq 1$. Now the above argument of uniqueness of $\widehat{\|\cdot\|}_{L, \bar{f}}$ implies $\widehat{\|\cdot\|}_{L, \bar{f}}^{\prime \prime}=\widehat{\|\cdot\|}_{L, \bar{f}}$. This completes the proof.

In the rest of this section, we consider a case $K_{v}=\mathbb{C}, X=\mathbb{P}^{N}$ and $L=\mathcal{O}_{\mathbb{P}^{N}}(1)$. We endow $\mathcal{O}_{\mathbb{P}^{N}}(1)$ with the Fubini-Study metric $\|\cdot\|_{F S}$.

In this case, to give $\bar{f}=(f, \varphi) \in \overline{\mathcal{H}}$ is equivalent to give homogeneous polynomials $F_{0}\left(X_{0}, \cdots, X_{N}\right), \ldots, F_{N}\left(X_{0}, \cdots, X_{N}\right) \in \mathbb{C}\left[X_{0}, \cdots, X_{N}\right]$ of degree $d_{f}$ such that $f=\left(F_{0}\right.$ : $\left.\cdots: F_{N}\right)$. Indeed $F_{k}=\varphi^{*} f^{*}\left(X_{k}\right)$, where $X_{k}$ is regarded as an element of $\Gamma\left(\mathbb{P}^{N}, \mathcal{O}_{\mathbb{P}^{N}}(1)\right)$. Note that, since $f: \mathbb{P}^{N} \rightarrow \mathbb{P}^{N}$ is a morphism, the only common zero of $F_{0}, \ldots, F_{N}$ is $0 \in \mathbb{C}^{N+1}$.

Let $\overline{\boldsymbol{f}}=\left(\overline{f_{i}}\right)_{i=1}^{\infty} \in \overline{\mathcal{B}}$. Let $F_{0}^{i}\left(X_{0}, \cdots, X_{N}\right), \ldots, F_{N}^{i}\left(X_{0}, \cdots, X_{N}\right) \in \mathbb{C}\left[X_{0}, \cdots, X_{N}\right]$ be homogeneous polynomials of degree $d_{f_{i}}$ corresponding to $\overline{f_{i}}$. We put, for $i \geq 1$,

$$
F^{i}:=\left(F_{0}^{i}, \cdots, F_{N}^{i}\right): \mathbb{C}^{N+1} \rightarrow \mathbb{C}^{N+1} .
$$

For $x=\left(x_{0}, \cdots, x_{N}\right) \in \mathbb{C}^{N+1}$, we put $|x|=\sqrt{\left|x_{0}\right|^{2}+\cdots+\left|x_{N}\right|^{2}}$.

We set $G_{0}(x)=\log |x|^{2}$, and for $i \geq 1$ we define $G_{i}: \mathbb{C}^{N+1} \backslash\{0\} \rightarrow \mathbb{R}$ by

$$
G_{i}(x)=\frac{1}{\prod_{\alpha=1}^{i} d_{f_{\alpha}}} \log \left|F^{i} \circ \cdots \circ F^{1}(x)\right|^{2} .
$$

Let $\mathcal{F}_{\bar{f}} \subset \mathbb{P}^{N}(\mathbb{C})$ be the largest open set on which the family $\left\{f_{1}, f_{2} \circ f_{1}, f_{3} \circ f_{2} \circ f_{1}, \cdots\right\}$ is normal, and $\mathcal{J}_{\bar{f}}$ be its complement.

Lemma 6.2. For any $i \geq 1$ and $x=\left(x_{0}, \cdots, x_{N}\right) \in \mathbb{C}^{N+1} \backslash\{0\}$, we have

$$
\left|G_{i}(x)-G_{i-1}(x)\right| \leq \frac{\bar{c}(\bar{f})}{2^{i-2}} .
$$


Proof. As in the proof of Theorem 6.1, we let $g_{i}=\log \frac{\left(\varphi_{i}^{*} f_{i}^{*}\|\cdot\|_{F S}\right)^{\frac{1}{d_{f_{i}}}}}{\|\cdot\|_{F S}}$. For $x=\left(x_{0}, \cdots, x_{N}\right) \in$ $\mathbb{C}^{N+1} \backslash\{0\}$ and $\pi(x)=\left(x_{0}: \cdots: x_{N}\right) \in \mathbb{P}^{N}(\mathbb{C})$, we auxiliary take $k$ such that $F_{k}^{i}(x) \neq 0$. We regard $F_{k}^{i}$ as an element of $\Gamma\left(\mathbb{P}^{N}, \mathcal{O}_{\mathbb{P}^{N}}\left(d_{f_{i}}\right)\right)$. Noting that $\varphi_{i}^{*} f_{i}^{*}\left(X_{k}\right)=F_{k}^{i}$, we then get

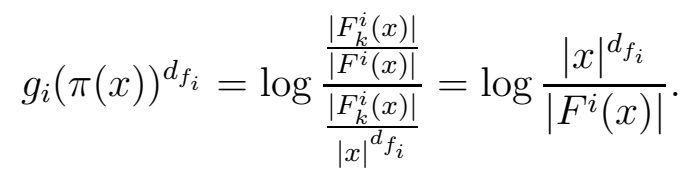

It follows from $\left|g_{i}(\pi(x))\right| \leq \bar{c}(\overline{\boldsymbol{f}})$ that

$$
\left|\frac{1}{d_{f_{i}}} \log \right| F^{i}(x)|-\log | x|| \leq \bar{c}(\bar{f}) .
$$

Then we have

$$
\left|\frac{1}{d_{f_{i}}} \log \right| F^{i}\left(F^{i-1} \circ \cdots \circ F^{1}(x)\right)|-\log | F^{i-1} \circ \cdots \circ F^{1}(x)|| \leq \bar{c}(\bar{f}) .
$$

Thus

$$
\left|G_{i}(x)-G_{i-1}(x)\right| \leq \frac{2 \bar{c}(\overline{\boldsymbol{f}})}{\prod_{\alpha=1}^{i-1} d_{f_{\alpha}}} \leq \frac{\bar{c}(\overline{\boldsymbol{f}})}{2^{i-2}}
$$

Proposition 6.3 (cf. [9]). Let $X=\mathbb{P}^{N}$ over $\mathbb{C},(L,\|\cdot\|)=\left(\mathcal{O}_{\mathbb{P}^{N}}(1),\|\cdot\|_{F S}\right)$, and $\overline{\boldsymbol{f}} \in \overline{\mathcal{B}}$.

(1) As $l$ tends to the infinity, $G_{l}$ converges uniformly to a plurisubharmonic and continuous function $G_{\bar{f}}: \mathbb{C}^{N+1} \backslash\{0\} \rightarrow \mathbb{R}$.

(2) Let $T_{\bar{f}}$ be the positive closed $(1,1)$ current on $\mathbb{P}^{N}(\mathbb{C})$ such that $\pi^{*} T_{\bar{f}}=d d^{c}\left(G_{\bar{f}}\right)$, where $\pi: \mathbb{C}^{N+1} \backslash\{0\} \rightarrow \mathbb{P}^{N}$ is the natural projection. Then $\operatorname{Supp}\left(T_{\overline{\boldsymbol{f}}}\right)=\mathcal{J}_{\overline{\boldsymbol{f}}}$.

We call $G_{\bar{f}}$ the Green function for $\overline{\boldsymbol{f}}$, and $T_{\bar{f}}$ the Green current for $\overline{\boldsymbol{f}}$.

Proof. Using Lemma 6.2, one can prove (1) as in [9, §2], and (2) as in [9, Proposition 8], [17, Théorème 1.6.5].

Let $\widehat{\|\cdot\|_{\mathcal{O}_{\mathbb{P}}}(1), \overline{\boldsymbol{f}}}$ be the admissible metric for $\bar{f}$ on $\mathcal{O}_{\mathbb{P}^{N}}(1)$ over $\mathbb{P}_{\mathbb{C}}^{N}$. We define the first Chern current of $\left(\mathcal{O}_{\mathbb{P}^{N}}(1), \widehat{\|\cdot\|_{\mathcal{O}^{N}}(1), \overline{\boldsymbol{f}}}\right)$ by

$$
c_{1}\left(\mathcal{O}_{\mathbb{P}^{N}}(1),{\widehat{\|\cdot\|_{\mathbb{P}^{N}}(1), \overline{\boldsymbol{f}}}}:=d d^{c}\left[-\log {\widehat{\|s\|_{\mathcal{O}^{N}(1)}, \overline{\boldsymbol{f}}}}^{2}+\delta_{\operatorname{div}(s)}\right.\right.
$$

for any nonzero section $s$ of $\mathcal{O}_{\mathbb{P}^{N}}(1)$. It is noted in [12, Proposition 3.3.1] that, when $f_{1}=$ $f_{2}=\cdots(=f)$, the first Chern current of $\mathcal{O}_{\mathbb{P}^{N}}(1)$ with the admissible metric coincides with the Green current. The following lemma says this also holds for $\bar{f} \in \overline{\mathcal{B}}$.

Proposition 6.4. With the notation and assumption as in Proposition 6.3, we have

$$
c_{1}\left(\mathcal{O}_{\mathbb{P}^{N}}(1), \widehat{\|\cdot\|_{\mathcal{O}^{N}}(1), \bar{f}}\right)=T_{\bar{f}} .
$$


Proof. Let $U_{0}:=\left\{\left(1: x_{1}: \cdots: x_{N}\right)\right\}$ be an open set of $\mathbb{P}^{N}(\mathbb{C})$. Since it suffices to prove the equality locally, we show it over $U_{0}$. (The case over $U_{i}:=\left\{\left(x_{0}: x_{1}: \cdots: x_{N}\right) \mid x_{i} \neq 0\right\}$ is proven similarly.) We take $\left(x_{1}, \cdots, x_{N}\right)$ as a coordinate of $U_{0}$. Then for $x=\left(1, x_{1}, \cdots, x_{N}\right) \in$ $\mathbb{C}^{N+1}$ and $\pi(x)=\left(1: x_{1}: \cdots: x_{N}\right) \in \mathbb{P}^{N}(\mathbb{C})$, we have

$$
\begin{aligned}
\log & \frac{\widehat{\|}_{\mathcal{O}_{\mathbb{P}^{N}}(1), \overline{\boldsymbol{f}}}^{2}}{\|\cdot\|_{F S}^{2}}(\pi(x))=2 \sum_{\alpha=1}^{\infty} \prod_{\beta=1}^{\alpha-1}\left(\frac{1}{d_{f_{\beta}}} \varphi_{\beta}^{*} f_{\beta}^{*}\right) g_{\alpha}(\pi(x)) \\
& =\sum_{\alpha=1}^{\infty} \frac{1}{\prod_{\beta=1}^{\alpha-1} d_{f_{\beta}}}\left(\log \left|F^{\alpha-1} \circ \cdots \circ F^{1}(x)\right|^{2}-\frac{1}{d_{f_{\alpha}}} \log \left|F^{\alpha} \circ F^{\alpha-1} \circ \cdots \circ F^{1}(x)\right|^{2}\right) \\
& =\sum_{\alpha=1}^{\infty}\left(G_{\alpha-1}(x)-G_{\alpha}(x)\right)=\log |x|^{2}-G_{\bar{f}}(x) .
\end{aligned}
$$

Taking $d d^{c}$ on both sides, we get the assertion.

We remark the following lemma.

Lemma 6.5. Let $X=\mathbb{P}^{N}$ over $\mathbb{C},(L,\|\cdot\|)=\left(\mathcal{O}_{\mathbb{P}^{N}}(1),\|\cdot\|_{F S}\right)$, and $\overline{\boldsymbol{f}}=\left(\left(f_{i}, \varphi_{i}\right)\right)_{i=0}^{\infty}, \overline{\boldsymbol{f}}^{\prime}=$ $\left(\left(f_{i}^{\prime}, \varphi_{i}^{\prime}\right)\right)_{i=0}^{\infty} \in \overline{\mathcal{B}}$. If $f_{i}=f_{i}^{\prime}$ for all $i$, then $T_{\bar{f}}=T_{\bar{f}^{\prime}}$

Proof. Let $F^{i^{\prime}}=\left(F_{0}^{i^{\prime}}, \cdots, F_{N}^{i^{\prime}}\right): \mathbb{C}^{N+1} \rightarrow \mathbb{C}^{N+1}$ be the lift of $f_{i}^{\prime}: \mathbb{P}^{N} \rightarrow \mathbb{P}^{N}$ given by $\varphi_{i}^{\prime}$. Then $F^{i^{\prime}}=F^{i}$ for some non-zero constant $c_{i} \in \mathbb{C}$. Then

$$
\begin{aligned}
G_{i}^{\prime}(x) & =\frac{1}{\prod_{\alpha=1}^{i} d_{f_{\alpha}}} \log \left|F^{i^{\prime}} \circ \cdots \circ F^{1^{\prime}}(x)\right|^{2} \\
& =\frac{1}{\prod_{\alpha=1}^{i} d_{f_{\alpha}}} \log \left|F^{i} \circ \cdots \circ F^{1}(x)\right|^{2}+\frac{1}{\prod_{\alpha=1}^{i} d_{f_{\alpha}}} \log \left|c_{1}^{d_{f_{i}} \cdots d_{f_{2}}} c_{2}^{d_{f_{i}} \cdots d_{f_{3}}} \cdots c_{i-1}^{d_{f_{i}}}\right|^{2} \\
& =G_{i}(x)+\sum_{k=1}^{i-1} \frac{1}{\prod_{\beta=1}^{k} d_{f_{\beta}}} \log \left|c_{k}\right|^{2} .
\end{aligned}
$$

By Proposition 6.3(1), we get $G_{\bar{f}}=G_{\bar{f}^{\prime}}+\sum_{k=1}^{\infty} \frac{1}{\prod_{\beta=1}^{k} d_{f_{\beta}}} \log \left|c_{k}\right|^{2}$ (The last term converges). Then Proposition 6.3(2) yields the lemma.

\section{Equidistribution of SMALL POINTS ON $\mathbb{P}^{1}$}

In this section, we show equidistribution of small points on $\mathbb{P}^{1}$ for sequences of morphisms. Let us first recall some facts on analytic torsions. For the details, we refer to [16].

Let $(M, k)$ be a $d$-dimensional compact Kähler manifold with the Kähler metric $k$. Let $(L, h)$ a $C^{\infty}$ hermitian line bundle over $M$. The vector space $A^{0, q}\left(L^{\otimes n}\right)$ of smooth $(0, q)$ forms on $M$ with values in $L^{\otimes n}$ is equipped with the $L^{2}$-metric given by

$$
(s, t)_{L^{2}}=\frac{1}{(2 \pi)^{d}} \int_{M}\langle s(x), t(x)\rangle d v \quad\left(s, t \in A^{0, q}\left(L^{\otimes n}\right)\right),
$$

where $d v$ is the normalized volume form on $M$ associated with $k$. Let $\bar{\partial}: A^{0, q}\left(L^{\otimes n}\right) \rightarrow$ $A^{0, q+1}\left(L^{\otimes n}\right)$ be the Dolbeault operator and $\bar{\partial}^{*}: A^{0, q}\left(L^{\otimes n}\right) \rightarrow A^{0, q-1}\left(L^{\otimes n}\right)$ its formal adjoint 
with respect to the $L^{2}$-metric. Let $\square_{n}^{q}:=\left(\bar{\partial}+\bar{\partial}^{*}\right)^{2}$ be the Laplacian acting on $A^{0, q}\left(L^{\otimes n}\right)$. Let $\sigma\left(\square_{n}^{q}\right)$ be the spectrum of $\square_{n}^{q}$. We set, for $s \in \mathbb{C}$,

$$
\zeta_{q, n}(s):=\sum_{\lambda \in \sigma\left(\square_{n}^{q}\right) \backslash\{0\}} \lambda^{-s} .
$$

The zeta function $\zeta_{q, n}(s)$ converges absolutely for $\operatorname{Re} s>d$, extends meromorphically to the whole plane, and is holomorphic at $s=0$. Then (the twice of the logarithm) of the Ray-Singer analytic torsion $T\left(L^{\otimes n}\right)$ is defined by, e.g., [16, p. 132],

$$
T\left(L^{\otimes n}\right)=\sum_{q=0}^{d}(-1)^{q+1} q \zeta_{q, n}^{\prime}(0) .
$$

Vojta [19, Propositon 2.7.6] proved the following theorem (see also Bismut-Vasserot [4]). We remark that in [19], $\tau\left(E_{\sigma}\right)$ is defined by $\tau\left(E_{\sigma}\right)=-T\left(E_{\sigma}\right)$.

Theorem $7.1([19])$. For each $q(0 \leq q \leq d)$, there exists a constant $C$ such that, as $n \rightarrow \infty$,

$$
\zeta_{q, n}^{\prime}(0) \geq-C n^{d} \log n .
$$

If $d=1$ then $T\left(L^{\otimes n}\right)=\zeta_{1, n}^{\prime}(0)$. Hence, if $M$ is a compact Riemann surface, then

$$
T\left(L^{\otimes n}\right) \geq-C n \log n .
$$

Question 7.2. Let $(M, k)$ be a $d$-dimensional compact Kähler manifold with the Kähler metric $k$, and $L$ a positive line bundle over $M$. Let $h$ be an arbitrary $C^{\infty}$ metric on $L$. (We do not assume $\mathrm{c}_{1}(L, h)$ is positive). Then is it true that, for any positive $\varepsilon$, there is $n_{0}$ such that $T\left(L^{\otimes n}\right) \geq-\varepsilon n^{d+1}$ for all $n \geq n_{0}$ ?

It follows from (7.1) that Question 7.2 is true for $d=1$. For any $d \geq 1$, Bismut-Vasserot [4] showed that if $\mathrm{c}_{1}(L, h)$ is positive then $T\left(L^{\otimes n}\right)=O\left(n^{d} \log d\right)$. They also showed that $T\left(L^{\otimes n}\right)=O\left(n^{d+1}\right)$ for an arbitrary $C^{\infty}$ metric $h([4$, Theorem 11]). Question 7.2 seems interesting, because the validity of Question 7.2 for $d=1$ is the key to prove equidistribution of small points on $\mathbb{P}^{1}$ in Theorem 7.5 below.

Before going back to sequences of morphisms, we need some results on arithmetic surfaces.

Let $K$ be a number field, and $O_{K}$ its ring of integers. Let $\mathcal{X}$ be a projective arithmetic surface (i.e., a projective arithmetic variety of dimension 2$)$, and $\overline{\mathcal{L}}=\left(\mathcal{L},\left\{\|\cdot\|_{\sigma}\right\}_{\sigma}\right)$ a $C^{\infty}$ hermitian line bundle.

We set $\Gamma=H^{0}(\mathcal{X}, \mathcal{L})$. Then $\Gamma$ is a lattice of $V:=H^{0}(\mathcal{X}, \mathcal{L}) \otimes_{\mathbb{Z}} \mathbb{R}$. By fixing an isomorphism $V \simeq \mathbb{R}^{\oplus \operatorname{rk} \Gamma}$, we give the Lebesgue measure $\mu$ on $V$. Since $V$ is the complex-conjugationinvariant subspace of $H^{0}(\mathcal{X}, \mathcal{L}) \otimes_{\mathbb{Z}} \mathbb{C}$, the metrics $\left\{\|\cdot\|_{\sigma}\right\}_{\sigma}$ induces the $L^{2}$-norm and sup norm on $V$. We set $\operatorname{vol}_{L^{2}}(\Gamma)=\frac{\mu(V / \Gamma)}{\mu\left(B_{L^{2}}(V)\right)}$ and $\operatorname{vol}_{\text {sup }}(\Gamma)=\frac{\mu(V / \Gamma)}{\mu\left(B_{\text {sup }}(V)\right)}$, where $B_{L^{2}}(V)=\{x \in V \mid$ $\left.\|x\|_{L^{2}} \leq 1\right\}$ and $B_{\text {sup }}(V)=\left\{x \in V \mid\|x\|_{\text {sup }} \leq 1\right\}$. The arithmetic Euler characteristics $\chi_{L^{2}}(\mathcal{X}, \overline{\mathcal{L}})$ and $\chi_{\text {sup }}(\mathcal{X}, \overline{\mathcal{L}})$ are then respectively defined by

$$
\chi_{L^{2}}(\mathcal{X}, \overline{\mathcal{L}})=-\log \operatorname{vol}_{L^{2}}(\Gamma) \quad \text { and } \quad \chi_{\text {sup }}(\mathcal{X}, \overline{\mathcal{L}})=-\log \operatorname{vol}_{\text {sup }}(\Gamma) .
$$

Theorem 7.3. Let $\mathcal{X}$ be a projective arithmetic surface over $\operatorname{Spec}\left(O_{K}\right)$, and $\overline{\mathcal{L}}=\left(\mathcal{L},\left\{\|\cdot\|_{\sigma}\right\}_{\sigma}\right)$ a $C^{\infty}$ hermitian line bundle on $\mathcal{X}$. Assume $\mathcal{L}$ is ample on $\mathcal{X}$. 
(1) If $\widehat{\operatorname{deg}}\left(\widehat{c}_{1}(\overline{\mathcal{L}})^{2}\right)>0$, then there is a constant $C$ such that, as $n \rightarrow \infty$,

$$
\chi_{\text {sup }}\left(\mathcal{X}, \overline{\mathcal{L}}^{\otimes n}\right) \geq \frac{\widehat{\operatorname{deg}}\left(\widehat{\mathrm{c}}_{1}(\overline{\mathcal{L}})^{2}\right)}{2} n^{2}+C n \log n .
$$

(2) For any $\varepsilon>0$, there exist a positive integer $n$ and a non-zero section $s \in H^{0}\left(\mathcal{X}, \mathcal{L}^{\otimes n}\right)$ such that

$$
\|s\|_{\sigma, \text { sup }} \leq \exp \left(n\left(\varepsilon-\frac{\widehat{\operatorname{deg}}\left(\widehat{\mathrm{c}}_{1}(\overline{\mathcal{L}})^{2}\right)}{2[K: \mathbb{Q}] \operatorname{deg}\left(\mathcal{L} \otimes_{O_{K}} K\right)}\right)\right)
$$

for all embeddings $\sigma: K \hookrightarrow \mathbb{C}$.

Proof. If $\mathrm{c}_{1}\left(\overline{\mathcal{L}} \otimes_{O_{K}^{\sigma}} \mathbb{C}\right)$ is positive for all $\sigma$, then Theorem 7.3 is just a very special case of [10, Theorem 8, Theorem 9], [16, Chap. VIII]. Using (7.1), one can prove Theorem 7.3 as in op. cit. For the sake of completeness, we sketch a proof here.

(1) Let $B_{r}$ denote the unit ball in $\mathbb{R}^{r}$ with the Euclidean metric. We have, for large $n$,

$$
\begin{aligned}
\chi_{\sup }\left(\mathcal{X}, \overline{\mathcal{L}}^{\otimes n}\right) & \chi_{L^{2}}\left(\mathcal{X}, \overline{\mathcal{L}}^{\otimes n}\right)+O(n \log n) \\
= & \widehat{\operatorname{deg}}\left(\widehat{\mathrm{c}}_{1}\left(\lambda\left(\overline{\mathcal{L}}^{\otimes n}\right), \text { Quillen metric }\right)\right)-\sum_{q=1}^{2}(-1)^{q} \log \# H^{q}\left(\mathcal{X}, \mathcal{L}^{\otimes n}\right) \\
& \quad+\log \operatorname{vol}\left(B_{\mathrm{rk}} H^{0}\left(\mathcal{X}, \mathcal{L}^{\otimes n}\right)\right)+\frac{1}{2} T\left(\mathcal{L}^{\otimes n} \otimes_{\mathbb{Z}} \mathbb{C}\right)+O(n \log n) \\
= & \frac{\widehat{\operatorname{deg}}\left(\widehat{\mathrm{c}}_{1}(\overline{\mathcal{L}})^{2}\right)}{2} n^{2}+\frac{1}{2} T\left(\mathcal{L}^{\otimes n} \otimes_{\mathbb{Z}} \mathbb{C}\right)+O(n \log n) \\
\geq & \frac{\widehat{\operatorname{deg}}\left(\widehat{\mathrm{c}}_{1}(\overline{\mathcal{L}})^{2}\right)}{2} n^{2}+C n \log n .
\end{aligned}
$$

Here we used the $L^{2}$-sup comparison due to Gromov (cf. [10, Lemma 30]) in the first equality, the definition of Quillen metrics (cf. [16, Chap. VIII. Lemma 1], [10, §4]) in the second equality, Gillet-Soulé's arithmetic Riemann-Roch theorem (cf. [10, Theorem 7], [16, Chap. VIII]) in the third equality, and Vojta's estimate (7.1) in the last inequality.

(2) Let $\varepsilon$ be any positive number. Let $\overline{\mathcal{L}}^{\prime}$ be the $\mathbb{Q}$-line bundle $\mathcal{L}$ on $\mathcal{X}$ equipped with the metric $\|\cdot\|_{\sigma}^{\prime}$ given by

$$
\|\cdot\|_{\sigma}^{\prime}=\|\cdot\|_{\sigma} \exp \left(\frac{\widehat{\operatorname{deg}}\left(\widehat{\mathrm{c}}_{1}(\overline{\mathcal{L}})^{2}\right)}{2[K: \mathbb{Q}] \operatorname{deg}\left(\mathcal{L} \otimes_{O_{K}} K\right)}-\varepsilon\right)
$$

for all $\sigma: K \hookrightarrow \mathbb{C}$. Then

$$
\begin{aligned}
\widehat{\operatorname{deg}}\left(\widehat{\mathrm{c}}_{1}\left(\overline{\mathcal{L}^{\prime}}\right)^{2}\right) & =\widehat{\operatorname{deg}}\left(\widehat{\mathrm{c}}_{1}(\overline{\mathcal{L}})^{2}\right)+2[K: \mathbb{Q}] \operatorname{deg}\left(\mathcal{L} \otimes_{O_{K}} K\right)\left(\varepsilon-\frac{\widehat{\operatorname{deg}}\left(\widehat{\mathrm{c}}_{1}(\overline{\mathcal{L}})^{2}\right)}{2[K: \mathbb{Q}] \operatorname{deg}\left(\mathcal{L} \otimes_{O_{K}} K\right)}\right) \\
& =2[K: \mathbb{Q}] \operatorname{deg}\left(\mathcal{L} \otimes_{O_{K}} K\right) \varepsilon>0 .
\end{aligned}
$$

We set $\Gamma_{n}=H^{0}\left(\mathcal{X}, \mathcal{L}^{\otimes n}\right)$ and $V_{n}=H^{0}\left(\mathcal{X}, \mathcal{L}^{\otimes n}\right) \otimes_{\mathbb{Z}} \mathbb{R}$. We endow $V_{n}$ with the sup metric $\|\cdot\|_{\text {sup }}^{\prime}$ induced by $\left\{\|\cdot\|_{\sigma}^{\prime n}\right\}_{\sigma}$. We set $\lambda_{1}\left(\Gamma_{n}\right)=\left\{\|s\|_{\text {sup }}^{\prime} \mid 0 \neq s \in \Gamma_{n}\right\}$. We also set $\operatorname{vol}_{\text {sup }}\left(\Gamma_{n}\right)=$ 
$\frac{\mu\left(V_{n} / \Gamma_{n}\right)}{\mu\left(B_{\sup }\left(V_{n}\right)\right)}$, where $\mu$ is a Lebesgue measure on $V_{n}$ and $B_{\text {sup }}\left(V_{n}\right)=\left\{x \in V_{n} \mid\|x\|_{\text {sup }}^{\prime} \leq 1\right\}$. Minkowski's convex body theorem states that

$$
\left(\operatorname{dim} V_{n}\right) \log \lambda_{1}\left(\Gamma_{n}\right) \leq\left(\operatorname{dim} V_{n}\right) \log 2+\log \operatorname{vol}_{\text {sup }}\left(\Gamma_{n}\right) .
$$

It follows from (1) that $\log \operatorname{vol}_{\text {sup }}\left(\Gamma_{n}\right) \leq-\frac{\widehat{\operatorname{deg}}\left(\widehat{\mathrm{c}}_{1}\left(\overline{\mathcal{L}}^{\prime}\right)^{2}\right)}{2} n^{2}-C n \log n$ for large $n$. On the other hand, $\operatorname{dim} V_{n}=[K: \mathbb{Q}]\left\{\operatorname{deg}\left(\mathcal{L} \otimes_{O_{K}} K\right) n+\left(1-g\left(\mathcal{X}_{\sigma}(\mathbb{C})\right)\right)\right\}$ for large $n$. Hence, for any positive $\varepsilon^{\prime}>0$, we find $\log \lambda_{1}\left(\Gamma_{n}\right) \leq n\left(\varepsilon^{\prime}-\frac{\widehat{\operatorname{deg}}\left(\widehat{\mathrm{c}}_{1}\left(\overline{\mathcal{L}}^{\prime}\right)^{2}\right)}{2[K: \mathbb{Q}] \operatorname{deg}\left(\mathcal{L}_{O_{K} K}\right)}\right)$ for large $n$. Taking $\varepsilon^{\prime}=\varepsilon$, we see $\log \lambda_{1}\left(\Gamma_{n}\right) \leq 0$. Then the assertion follows from (7.2).

In what follows, we fix an embedding $\sigma_{0}: K \hookrightarrow \mathbb{C}$, and extend $\sigma_{0}$ to $\sigma_{0}: \bar{K} \hookrightarrow \mathbb{C}$.

We consider the case $X=\mathbb{P}^{1}$ and $L=\mathcal{O}_{\mathbb{P}^{1}}(1)$. For $i=1,2, \ldots$, let $f_{i}: \mathbb{P}^{1} \rightarrow \mathbb{P}^{1}$ be a surjective morphism over $K$ of degree $d_{f_{i}} \geq 2$. We define the morphism $f_{i \mathbb{C}}: \mathbb{P}_{\mathbb{C}}^{1} \rightarrow \mathbb{P}_{\mathbb{C}}^{1}$ over $\mathbb{C}$ by $f_{i \mathbb{C}}:=f_{i} \times_{K^{\sigma_{0}}} \mathbb{C}: \mathbb{P}^{1} \times_{K^{\sigma_{0}}} \mathbb{C} \rightarrow \mathbb{P}^{1} \times_{K^{\sigma_{0}}} \mathbb{C}$.

For $f:=\left(f_{i}\right)_{i=1}^{\infty}$, we assume the following two conditions:

$(\mathrm{C}-\mathrm{i}) \boldsymbol{f}$ is adelically bounded such that $\mathcal{L}_{0}$ is ample on $\mathcal{X}_{0}$ (cf. $\S 5$ );

(C-ii) There are isomorphisms $\varphi_{i}: \mathcal{O}_{\mathbb{P}_{\mathbb{C}}^{1}}\left(d_{f_{i}}\right) \rightarrow f_{i \mathbb{C}}^{*} \mathcal{O}_{\mathbb{P}_{\mathbb{C}}^{1}}(1)$ over $\mathbb{C}$ for $i=1,2, \ldots$ such that

$$
\sup _{i \geq 1} \sup _{x \in \mathbb{P}^{1}(\mathbb{C})}\left|\log \frac{\left(\varphi_{i}^{*} f_{i \mathbb{C}}^{*}\|\cdot\|_{F S}\right)^{\frac{1}{d_{f_{i}}}}}{\|\cdot\|_{F S}}(x)\right|<+\infty,
$$

where $\|\cdot\|_{F S}$ is the Fubini-Study metric on $\mathcal{O}_{\mathbb{P}_{\mathbb{C}}^{1}}(1)$ over $\mathbb{P}_{\mathbb{C}}^{1}$.

For example, iterations by a finite number of morphisms $g_{1}, \ldots, g_{k}$ in Example 2.1 satisfy these conditions. For $f$ satisfying $(\mathrm{C}-\mathrm{i})$ and $(\mathrm{C}-\mathrm{ii})$, it follows from Proposition 5.5 and Proposition 6.3 that one has the canonical height function $\widehat{h}_{\mathbb{P}^{1}, f}: \mathbb{P}^{1}(\bar{K}) \rightarrow \mathbb{R}$ and the Green current $T_{\boldsymbol{f}}$ on $\mathbb{P}^{1}(\mathbb{C})$. Here, in virtue of Lemma 6.5 , we use the notation $T_{\boldsymbol{f}}$ instead of $T_{\overline{\boldsymbol{f}}}$, where $\bar{f}=\left(\left(f_{i}, \varphi_{i}\right)\right)_{i=1}^{\infty}$.

Let $\left(x_{j}\right)_{j=1}^{\infty}$ be a sequence with $x_{j} \in \mathbb{P}^{1}(\bar{K})$. The sequence $\left(x_{j}\right)_{j=1}^{\infty}$ is said to be generic if, for any $x \in \mathbb{P}^{1}(\bar{K})$, there exists $j_{0}$ such that $x_{j} \neq x$ for any $j \geq j_{0}$. The sequence $\left(x_{j}\right)_{j=1}^{\infty}$ is called a sequence of small points with respect to $\widehat{h}_{\boldsymbol{f}}$ if $\lim _{j \rightarrow \infty} \widehat{h}_{\boldsymbol{f}}\left(x_{j}\right)=0$.

For $x \in \mathbb{P}^{1}(\bar{K})$, let $G(x):=\{\operatorname{Gal}(\bar{K} / K) \cdot x\} \subset \mathbb{P}^{1}(\bar{K})$ be the orbit of $x$ under the Galois group. For $y \in \mathbb{P}^{1}(\bar{K})$, let $\delta_{y}$ denote the Dirac measure of mass 1 on $\mathbb{P}^{1}(\mathbb{C})$ supported in $y$, where we regard $y$ as a point of $\mathbb{P}^{1}(\mathbb{C})$ through the embedding $\sigma_{0}: \bar{K} \hookrightarrow \mathbb{C}$.

Example 7.4. We fix $a \in \mathbb{P}^{1}(\bar{K})$ with $\widehat{h}_{\boldsymbol{f}}(a) \neq 0$. Let $x_{j} \in \mathbb{P}^{1}(\bar{K})$ be a point with $f_{j} \circ$ $f_{j-1} \circ \cdots \circ f_{1}\left(x_{j}\right)=a$. Since $\widehat{h}_{\boldsymbol{f}}\left(x_{j}\right)=\frac{1}{d_{f_{1} \cdots d_{f_{j}}}} \widehat{h}_{\boldsymbol{f}}(a), x_{j}$ 's are distinct points such that $\lim _{j \rightarrow \infty} \widehat{h}_{\boldsymbol{f}}\left(x_{j}\right)=0$. Thus $\left(x_{j}\right)_{j=1}^{\infty}$ is a generic sequence of small points with respect to $\widehat{h}_{\boldsymbol{f}}$.

Now we prove the following theorem on equidistribution of small points on $\mathbb{P}^{1}$ for $f$. We remark that this theorem will also follow from Autissier [1, Proposition 4.1.4 and Remarque in Introduction] (together with Proposition 5.5). We also remark that Baker-Hsia [2] proved equidistribution of small points on $\mathbb{P}^{1}$ for polynomial maps $f$ over global fields satisfying the product formula. 
Theorem 7.5. Let $\boldsymbol{f}=\left(f_{i}\right)_{i=1}^{\infty}$ be a sequence of morphisms of $\mathbb{P}^{1}$ over $K$ satisfying (Ci) and (C-ii). Let $\left(x_{j}\right)_{j=1}^{\infty}$ a generic sequence of small points with respect to $\widehat{h}_{\boldsymbol{f}}$. Then $\frac{1}{\# G\left(x_{j}\right)} \sum_{y \in G\left(x_{j}\right)} \delta_{y}$ converges weakly to $T_{f}$ as $j \rightarrow+\infty$.

Proof. We adapt the proof of [18]. It suffices to show that, for any $C^{\infty}$ real-valued function $\varphi$ on $\mathbb{P}^{1}(\mathbb{C})$, one has

$$
\lim _{j \rightarrow \infty} \frac{1}{\# G\left(x_{j}\right)} \sum_{y \in G\left(x_{j}\right)} \varphi(y)=T_{\boldsymbol{f}}(\varphi) .
$$

Let $\left\{\left(\mathcal{X}_{i}, \overline{\mathcal{L}}_{i}\right)\right\}_{i=0}^{\infty}$ be the adelic sequence associated with $\boldsymbol{f}$ in Proposition 5.3. We remark that $\mathcal{L}_{i}$ is ample on $\mathcal{X}_{i}$ because the normalization $f_{i U} \circ \cdots \circ f_{1 U}: \mathbb{P}_{U}^{1} \rightarrow \mathbb{P}_{U}^{1} \hookrightarrow \mathbb{P}_{O_{K}}^{1}$ is finite and $\mathcal{L}_{0}$ is assumed to be ample on $\mathcal{X}_{0}$. For $\lambda \in \mathbb{R}$, we define the hermitian $\mathbb{Q}$-line bundle $\overline{\mathcal{L}}_{i}(\lambda \varphi)$ to be the $\mathbb{Q}$-line bundle $\mathcal{L}_{i}$ on $\mathcal{X}_{i}$ equipped with the metrics $\left\{\|\cdot\|_{\sigma}^{\prime}\right\}_{\sigma}$ as follows: For all embeddings $\sigma$ except for $\sigma_{0}$ (resp. $\sigma_{0}$ and $\overline{\sigma_{0}}$ ) if $\sigma_{0}$ is real (resp. imaginary), the metric $\|\cdot\|_{\sigma}^{\prime}$ is the original metric $\|\cdot\|_{\sigma}$ of $\overline{\mathcal{L}}_{i}$; For the embedding $\sigma_{0}$ (resp. the embeddings $\sigma_{0}$ and $\left.\overline{\sigma_{0}}\right)$, the metric $\|\cdot\|_{\sigma}^{\prime}$ is defined by

$$
\begin{aligned}
& \|\cdot\|_{\sigma_{0}}^{\prime}=\|\cdot\|_{\sigma_{0}} \exp (-\lambda \varphi) \\
& \left(\text { resp. }\|\cdot\|_{\sigma_{0}}^{\prime}=\|\cdot\|_{\sigma_{0}} \exp (-\lambda \varphi) \text { and }\|\cdot\|_{\overline{\sigma_{0}}}^{\prime}=\|\cdot\|_{\overline{\sigma_{0}}} \exp (-\lambda \varphi)\right) .
\end{aligned}
$$

Claim 7.5.1. $\liminf _{j \rightarrow \infty} h_{\left(\mathcal{X}_{i}, \overline{\mathcal{L}}_{i}(\lambda \varphi)\right)}\left(x_{j}\right) \geq h_{\left(\mathcal{X}_{i}, \overline{\mathcal{L}}_{i}(\lambda \varphi)\right)}\left(\mathbb{P}^{1}\right)$.

To see the claim, let $\varepsilon$ be any positive real number. Since $\operatorname{deg}\left(\mathcal{L}_{i} \otimes_{O_{K}} K\right)=1$, it follows from Theorem 7.3 that there are a positive integer $n$ and a non-zero section $s \in H^{0}\left(\mathcal{X}_{i}, \mathcal{L}_{i}^{\otimes n}\right)$ such that

$$
\|s\|_{\sigma, \text { sup }}^{\prime} \leq \exp \left(n\left(\varepsilon-\frac{\widehat{\operatorname{deg}}\left(\widehat{\mathrm{c}}_{1}\left(\overline{\mathcal{L}}_{i}(\lambda \varphi)\right)^{2}\right)}{2[K: \mathbb{Q}]}\right)\right)
$$

for all embeddings $\sigma: K \hookrightarrow \mathbb{C}$.

Let $K_{j}$ be an extension field of $K$ such that $x_{j}$ is defined, and $O_{K_{j}}$ the ring of integers of $K_{j}$. Let $\Delta_{x_{j}}$ be the Zariski closure of $x_{j}$ in $\mathcal{X}_{i} \times_{\operatorname{Spec}\left(O_{K}\right)} \operatorname{Spec}\left(O_{K_{j}}\right)$. Let $p_{j}: \mathcal{X}_{i} \times_{\operatorname{Spec}\left(O_{K}\right)}$ $\operatorname{Spec}\left(O_{K_{j}}\right) \rightarrow \mathcal{X}_{i}$ be the natural morphism. Since $\left(x_{j}\right)_{j=0}^{\infty}$ is a generic sequence, we have $\Delta_{x_{j}} \not \subseteq \operatorname{Supp}\left(\operatorname{div}\left(p_{j}^{*} s\right)\right)$ for all $j \gg 1$. Then, for all $j \gg 1$, we get

$$
\begin{aligned}
h_{\left(\mathcal{X}_{i}, \overline{\mathcal{L}}_{i}(\lambda \varphi)\right)}\left(x_{j}\right) & =\frac{1}{\left[K_{j}: \mathbb{Q}\right]} \widehat{\operatorname{deg}}\left(\widehat{\mathrm{c}}_{1}\left(\left.p_{j}^{*} \overline{\mathcal{L}}_{i}(\lambda \varphi)\right|_{\Delta_{x_{j}}}\right)\right) \\
& \geq \frac{1}{\left[K_{j}: \mathbb{Q}\right]} \frac{1}{n}\left[K_{j}: K\right]\left(\sum_{\sigma: K \hookrightarrow \mathbb{C}}-\log \|s\|_{\sigma, \text { sup }}^{\prime}\right) \\
& \geq \frac{1}{\left[K_{j}: \mathbb{Q}\right]} \frac{1}{n}\left[K_{j}: K\right][K: \mathbb{Q}] n\left(\frac{\widehat{\operatorname{deg}}\left(\widehat{c}_{1}\left(\overline{\mathcal{L}}_{i}(\lambda \varphi)\right)^{2}\right)}{2[K: \mathbb{Q}]}-\varepsilon\right)=h_{\left(\mathcal{X}_{i}, \overline{\mathcal{L}_{i}}(\lambda \varphi)\right)}\left(\mathbb{P}^{1}\right)-\varepsilon
\end{aligned}
$$

Hence $\liminf _{j \rightarrow \infty} h_{\left(\mathcal{X}_{i}, \overline{\mathcal{L}}_{i}(\lambda \varphi)\right)}\left(x_{j}\right) \geq h_{\left(\mathcal{X}_{i}, \overline{\mathcal{L}}_{i}(\lambda \varphi)\right)}\left(\mathbb{P}^{1}\right)-\varepsilon$. Since $\varepsilon$ is arbitrary, we obtain the claim. 
Now we compute $h_{\left(\mathcal{X}_{i}, \overline{\mathcal{L}}_{i}(\lambda \varphi)\right)}\left(x_{j}\right)$ and $h_{\left(\mathcal{X}_{i}, \overline{\mathcal{L}}_{i}(\lambda \varphi)\right)}(X)$. We set $r=1$ if $\sigma_{0}$ is real and $r=2$ if $\sigma_{0}$ is imaginary. We have

$$
h_{\left(\mathcal{X}_{i}, \overline{\mathcal{L}}_{i}(\lambda \varphi)\right)}\left(x_{j}\right)=h_{\left(\mathcal{X}_{i}, \overline{\mathcal{L}}_{i}\right)}\left(x_{j}\right)+\frac{\lambda r}{[K: \mathbb{Q}]} \frac{1}{\# G\left(x_{j}\right)} \sum_{y \in G\left(x_{j}\right)} \varphi(y) .
$$

We also have

$$
\begin{aligned}
& h_{\left(\mathcal{X}_{i}, \overline{\mathcal{L}}_{i}(\lambda \varphi)\right)}\left(\mathbb{P}^{1}\right)=\frac{1}{2[K: \mathbb{Q}]} \widehat{\operatorname{deg}}\left(\widehat{\mathrm{c}}_{1}\left(\overline{\mathcal{L}}_{i}(\lambda \varphi)\right)^{2}\right) \\
& =\frac{1}{2[K: \mathbb{Q}]} \widehat{\operatorname{deg}}\left(\widehat{\mathrm{c}}_{1}\left(\overline{\mathcal{L}}_{i}\right)^{2}\right)+\frac{\lambda r}{[K: \mathbb{Q}]} \int_{\mathbb{P}^{1}(\mathbb{C})} \varphi c_{1}\left(\overline{\mathcal{L}}_{i} \otimes_{K^{\sigma}} \mathbb{C}\right)+\frac{\lambda^{2} r}{[K: \mathbb{Q}]} \int_{\mathbb{P}^{1}(\mathbb{C})} \varphi d d^{c}(\varphi) \\
& =h_{\left(\mathcal{X}_{i}, \overline{\mathcal{L}}_{i}\right)}\left(\mathbb{P}^{1}\right)+\frac{\lambda r}{[K: \mathbb{Q}]} \int_{\mathbb{P}^{1}(\mathbb{C})} \varphi c_{1}\left(\overline{\mathcal{L}}_{i} \otimes_{K^{\sigma}} \mathbb{C}\right)+\frac{\lambda^{2} r}{[K: \mathbb{Q}]} \int_{\mathbb{P}^{1}(\mathbb{C})} \varphi d d^{c}(\varphi) .
\end{aligned}
$$

It follows from Claim 7.5.1, (7.3) and (7.4) that

$$
\begin{gathered}
\liminf _{j \rightarrow \infty}\left(h_{\left(\mathcal{X}_{i}, \overline{\mathcal{L}}_{i}\right)}\left(x_{j}\right)+\frac{\lambda r}{[K: \mathbb{Q}]} \frac{1}{\# G\left(x_{j}\right)} \sum_{y \in G\left(x_{j}\right)} \varphi(y)\right) \\
\geq h_{\left(\mathcal{X}_{i}, \overline{\mathcal{L}}_{i}\right)}\left(\mathbb{P}^{1}\right)+\frac{\lambda r}{[K: \mathbb{Q}]} \int_{\mathbb{P}^{1}(\mathbb{C})} \varphi \mathrm{c}_{1}\left(\overline{\mathcal{L}}_{i} \otimes_{K^{\sigma_{0}}} \mathbb{C}\right)+\frac{\lambda^{2} r}{[K: \mathbb{Q}]} \int_{\mathbb{P}^{1}(\mathbb{C})} \varphi d d^{c}(\varphi) .
\end{gathered}
$$

Now we let $i$ to the infinity. Since the convergence $\widehat{h}_{\mathcal{O}_{\mathbb{P}}(1), \boldsymbol{f}}(x)=\lim _{i \rightarrow \infty} h_{\left(\mathcal{X}_{i}, \overline{\mathcal{L}}_{i}\right)}(x)$ is uniform with respect to $x \in \mathbb{P}^{1}(\bar{K})$ by Proposition 5.5 , we have

$$
\lim _{i \rightarrow \infty} \liminf _{j \rightarrow \infty} h_{\left(\mathcal{X}_{i}, \overline{\mathcal{L}}_{i}\right)}\left(x_{j}\right)=\liminf _{j \rightarrow \infty} \lim _{i \rightarrow \infty} h_{\left(\mathcal{X}_{i}, \overline{\mathcal{L}}_{i}\right)}\left(x_{j}\right)=\liminf _{j \rightarrow \infty} \widehat{h}_{\mathcal{O}_{\mathbb{P}} 1}(1), f\left(x_{j}\right)=0 .
$$

By Theorem 4.2(2)(iv) and Proposition 5.5, we get $\lim _{i \rightarrow \infty} h_{\left(\mathcal{X}_{i}, \overline{\mathcal{L}}_{i}\right)}\left(\mathbb{P}^{1}\right)=\widehat{h}_{\mathcal{O}_{\mathbb{P}}, f}\left(\mathbb{P}^{1}\right)=0$. Moreover, by Lemma 6.2, Proposition 6.4 and its proof, $c_{1}\left(\overline{\mathcal{L}}_{i} \otimes_{K^{\sigma_{0}}} \mathbb{C}\right)$ converges weakly to $T_{\boldsymbol{f}}$. Hence we get

$$
\liminf _{j \rightarrow \infty} \frac{\lambda r}{[K: \mathbb{Q}]} \frac{1}{\# G\left(x_{j}\right)} \sum_{y \in G\left(x_{j}\right)} \varphi(y) \geq \frac{\lambda r}{[K: \mathbb{Q}]} T_{\boldsymbol{f}}(\varphi)+\frac{\lambda^{2} r}{[K: \mathbb{Q}]} \int_{\mathbb{P}^{1}(\mathbb{C})} \varphi d d^{c}(\varphi) .
$$

Since $\lambda$ is an arbitrary real number, we find $\lim _{j \rightarrow \infty} \frac{1}{\# G\left(x_{j}\right)} \sum_{y \in G\left(x_{j}\right)} \varphi(y)=T_{\boldsymbol{f}}(\varphi)$. This completes the proof.

\section{REFERENCES}

[1] P. Autissier, Points entiers sur les surfaces arithmétiques, J. Reine Angew. Math. 531 (2001), 201-235.

[2] M. Baker and L.-C. Hsia, Canonical heights, transfinite diameters, and polynomial dynamics, preprint, math.NT/0305181.

[3] M. Baker and R. Rumely, Equidistribution of small points, rational dynamics, and potential theory, preprint, math.NT/0407426. 
[4] J.-M. Bismut and É. Vasserot, The asymptotics of the Ray-Singer analytic torsion associated with high powers of a positive line bundle, Comm. Math. Phys. 125 (1989), 355-367.

[5] J.-B. Bost, H. Gillet and C. Soulé, Heights of projective varieties and positive Green forms, J. Amer. Math. Soc. 7 (1994), 903-1027.

[6] A. Chambert-Loir, Mesures et équidistribution sur les espaces de Berkovich, preprint, math.NT/0304023.

[7] G. Call and H. Silverman, Canonical heights on varieties with morphisms, Compositio Math. 89 (1993), 163-205.

[8] C. Favre and J. Rivera-Letelier Equidistribution des points de petite hauteur, preprint, math.NT/0407471.

[9] J. E. Fornæss and B. Weickert, Random iteration in $P^{k}$, Ergodic Theory Dynam. Systems 20 (2000), 1091-1109.

[10] H. Gillet and C. Soulé, An arithmetic Riemann-Roch theorem, Invent. Math. 110 (1992), 473-543.

[11] M. Hindry and J. H. Silverman, Diophantine geometry. An introduction, Springer, New York, 2000.

[12] S. Kawaguchi, Canonical heights, invariant currents, and dynamical eigensystems of morphisms for line bundles, preprint, math.NT/0405006.

[13] J.-C. Masseron, Points entiers de fractions rationnelles et points périodiques de plusieurs polynômes, Thèse, l'Université Paris VI (2001).

[14] A. Moriwaki, The canonical arithmetic height of subvarieties of an abelian variety over a finitely generated field, J. Reine Angew. Math. 530 (2001), 33-54.

[15] A. Russakovskii and M. Sodin, Equidistribution for sequences of polynomial mappings, Indiana Univ. Math. J. 44 (1995), 851-882.

[16] C. Soulé, Lectures on Arakelov geometry, With the collaboration of D. Abramovich, J.-F. Burnol and J. Kramer, Cambridge Univ. Press, Cambridge, 1992.

[17] N. Sibony, Dynamique des applications rationnelles de $\mathbb{P}^{k}$, Panoramas et Synthèses 8 (1999), 97-185.

[18] L. Szpiro, E. Ullmo, S. Zhang, Équirépartition des petits points, Invent. Math. 127 (1997), 337-347.

[19] P. Vojta, Siegel's theorem in the compact case, Ann. of Math. 133 (1991), 509-548.

[20] S. Zhang, Small points and adelic metrics, J. Algebraic Geom. 4 (1995), 281-300.

Department of Mathematics, Faculty of Science, Kyoto University, Kyoto, 606-8502, JAPAN

E-mail address: kawaguch@math.kyoto-u.ac.jp 